\title{
Testing the No-hair Theorem with Event Horizon Telescope Observations of Sagittarius A*
}

\section{Citation}

Broderick, Avery E., Tim Johannsen, Abraham Loeb, and Dimitrios Psaltis. 2014. "TESTING THE NO-HAIR THEOREM WITH EVENT HORIZON TELESCOPE OBSERVATIONS OF SAGITTARIUS A*." The Astrophysical Journal 784 (1): 7. https://doi.org/10.1088/0004-637x/784/1/7.

\section{Permanent link}

http://nrs.harvard.edu/urn-3:HUL.InstRepos:41393194

\section{Terms of Use}

This article was downloaded from Harvard University's DASH repository, and is made available under the terms and conditions applicable to Other Posted Material, as set forth at http:// nrs.harvard.edu/urn-3:HUL.InstRepos:dash.current.terms-of-use\#LAA

\section{Share Your Story}

The Harvard community has made this article openly available.

Please share how this access benefits you. Submit a story.

Accessibility 


\title{
TESTING THE NO-HAIR THEOREM WITH EVENT HORIZON TELESCOPE OBSERVATIONS OF SAGITTARIUS A*
}

\author{
Avery E. Broderick ${ }^{1,2}$, Tim Johannsen ${ }^{1,2,3,6}$, Abraham Loeb $^{4}$, and Dimitrios Psaltis ${ }^{5}$ \\ ${ }^{1}$ Perimeter Institute for Theoretical Physics, 31 Caroline Street North, Waterloo, ON N2L 2Y5, Canada \\ ${ }^{2}$ Department of Physics and Astronomy, University of Waterloo, 200 University Avenue West, Waterloo, ON N2L 3G1, Canada \\ ${ }^{3}$ Canadian Institute for Theoretical Astrophysics, 60 St. George Street, Toronto, ON M5S 3H8, Canada \\ ${ }^{4}$ Institute for Theory and Computation, Harvard University, Center for Astrophysics, 60 Garden Street, Cambridge, MA 02138, USA \\ 5 Astronomy and Physics Departments, University of Arizona, 933 North Cherry Street, Tucson, AZ 85721, USA \\ Received 2013 November 19; accepted 2013 December 8; published 2014 February 26
}

\begin{abstract}
The advent of the Event Horizon Telescope (EHT), a millimeter-wave very long baseline interferometric array, has enabled spatially resolved studies of the subhorizon-scale structure for a handful of supermassive black holes. Among these, the supermassive black hole at the center of the Milky Way, Sagittarius A* (Sgr A*), presents the largest angular cross section. Thus far, these studies have focused on measurements of the black hole spin and the validation of low-luminosity accretion models. However, a critical input in the analysis of EHT data is the structure of the black hole spacetime, and thus these observations provide the novel opportunity to test the applicability of the Kerr metric to astrophysical black holes. Here we present the first simulated images of a radiatively inefficient accretion flow (RIAF) around Sgr A* employing a quasi-Kerr metric that contains an independent quadrupole moment in addition to the mass and spin that fully characterize a black hole in general relativity. We show that these images can be significantly different from the images of an RIAF around a Kerr black hole with the same spin and demonstrate the feasibility of testing the no-hair theorem by constraining the quadrupolar deviation from the Kerr metric with existing EHT data. Equally important, we find that the disk inclination and spin orientation angles are robust to the inclusion of additional parameters, providing confidence in previous estimations assuming the Kerr metric based on EHT observations. However, at present, the limits on potential modifications of the Kerr metric remain weak.
\end{abstract}

Key words: accretion, accretion disks - black hole physics - Galaxy: center - gravitation - submillimeter: general - techniques: interferometric

Online-only material: color figures

\section{INTRODUCTION}

Black holes are commonly invoked to power a variety of energetic phenomena. Stellar mass black holes are one of the presumed engines that power gamma-ray bursts (Mészáros 2006) and lie at the hearts of X-ray binaries (van der Klis 2006). Supermassive black holes are implicated in active galactic nuclei (AGNs), powering both prodigious electromagnetic luminosities and outflows. In the latter role supermassive black holes are believed to significantly impact the structures that surround them on cosmological scales. This occurs both by moderating accretion of baryons into dark matter halos (i.e., feedback; for a review, see Fabian 2012) and by injecting material back into the intergalactic medium (e.g., magnetic fields; Furlanetto \& Loeb 2001). Thus, supermassive black holes play a crucial role in coupling the smallest (horizon) and largest (cosmological) scales in astronomy.

These processes are fundamentally associated with the interaction of black holes with surrounding matter on scales comparable to the horizon via accretion flows, winds, jets, etc. As such, a tremendous effort continues to be expended studying the nature of these astrophysical interactions (Narayan 2005; Vietri 2008; Meier 2012). Critical to these is the stage set by the black hole, on which the astrophysical dynamics unfolds. Thus, imprinted within the electromagnetic signatures is information regarding the gravitational properties of the black hole itself.

\footnotetext{
6 CITA National Fellow.
}

Inferring properties of black hole spacetimes through their gravitational impact on the dynamics of surrounding matter has been accomplished successfully in a number of contexts. Examples include mass measurements via stellar orbital dynamics in the Galactic center (Ghez et al. 2008; Gillessen et al. 2009a, $2009 \mathrm{~b}$ ) and spin measurements via relativistically broadened $\mathrm{Fe}$ $\mathrm{K} \alpha$ line measurements (see, e.g., Reynolds 2013) and thin-disk spectra (see, e.g., McClintock et al. 2013). The quasiperiodic oscillations present in the spectra of some X-ray binaries (see, e.g., Remillard \& McClintock 2006) and in some AGNs (Gierliński et al. 2008; Reis et al. 2012) present a tantalizing additional signature, although in the absence of a unique model for their generation they are currently ambiguous. In these efforts, general relativity is accepted as a prior.

Similar attempts to constrain the applicability of general relativity have also been made. These may take the form of ruling out specific alternative gravity theories or employing one of a growing set of phenomenological Kerr-like metrics (see, e.g., Psaltis 2008; Myrzakulov et al. 2013). These differ from the Kerr metric, although they are not necessarily associated with any particular modified theory of gravity and, instead, often encompass large classes of such alternative theories (e.g., Manko \& Novikov 1992; Collins \& Hughes 2004; Glampedakis \& Babak 2006; Vigeland \& Hughes 2010; Johannsen \& Psaltis 2011a; Vigeland et al. 2011; Johannsen 2013b). As with spin, a variety of observations have been brought to bear on the applicability of the Kerr metric, including relativistically broadened iron lines (Johannsen \& Psaltis 2013; Bambi 2013), variability (Johannsen 
\& Psaltis 2011b, 2013; Bambi 2012b), continuum accretion disk spectra (Bambi \& Barausse 2011; Krawczynski 2012; Bambi 2012a), and X-ray polarization (Krawczynski 2012). Generally, this is considerably more difficult due to the potential degeneracy between erroneous accretion physics, which itself is not fully understood, and deviations from general relativity.

With the advent of millimeter-wavelength very long baseline interferometry (mm-VLBI) it has become possible to spatially resolve the horizon-scale structure for a handful of supermassive black holes. This provides an additional probe of both the gravitational and accretion/outflow physics. When combined with preexisting spectral information, mm-VLBI has proven to be a strong constraint on the black hole properties and provides a potential means to break the degeneracy between gravitational physics and astrophysics.

The best candidate for horizon-resolving mm-VLBI observations is the supermassive black hole at the center of the Milky Way, associated with the bright radio source Sgr A*. Because of its proximity, Sgr A* subtends the largest angle on the sky of any known black hole: with estimates of the mass and distance of $(4.3 \pm 0.5) \times 10^{6} M_{\odot}$ and $8.3 \pm 0.4 \mathrm{kpc}$, respectively, the lensed image of Sgr A*'s horizon is $53 \pm 2 \mu \mathrm{as}^{7}$ (Ghez et al. 2008; Gillessen et al. 2009a, 2009b). This is well matched to the resolution of existing and future mm-VLBI experiments, which access angular scales of $\gtrsim 30$ and $\gtrsim 10 \mu$ as, respectively. In addition, the spectral energy density of Sgr A* peaks near millimeter wavelengths, implying that the surrounding accretion flow transitions from being optically thick to optically thin at these wavelengths. Hence, the horizon is likely to be visible.

Unlike AGNs, Sgr A* is clearly underluminous, with a bolometric luminosity of $10^{-9}$ in Eddington units. This implies that it is qualitatively different from many AGNs, although perhaps more similar to the remaining $90 \%$ of supermassive black holes that are not currently active. While uncertainty remains regarding the morphology of the emission region, the existing spectral and polarization data have resulted in a canonical set of components in all models for Sgr A*: a peaked (often approximated by a Maxwellian) electron distribution function with a power-law high-energy tail and nearly equipartition magnetic fields. This results in a wide variety of potential models for the structure and dynamics of the emission region (e.g., Narayan et al. 1998; Blandford \& Begelman 1999; Falcke \& Markoff 2000; Özel et al. 2000; Yuan et al. 2003; Loeb \& Waxman 2007). Nevertheless, the existing mm-VLBI observations have already exhibited a nontrivial agreement with the radiatively inefficient accretion flow (RIAF) models, suggesting that these form a credible set of models for the purposes of imaging currently (Broderick et al. 2009, 2011b; Huang et al. 2009; Dexter et al. 2010; Shcherbakov et al. 2012; Dexter \& Fragile 2013).

Here we simulate the first images of an RIAF model around a supermassive black hole not described by the Kerr metric and assess the ability of existing mm-VLBI observations of Sgr A* to constrain possible deviations from general relativity. We do this by exploring the consequences of the well-developed quasiKerr metric, a parameterized modification of the Kerr metric that contains an independent quadrupole moment in addition to the mass and spin of the black hole (Glampedakis \& Babak 2006). This metric provides one of the simplest frameworks for the study of the effects of a modified quadrupole moment and parameterizes such deviations in a generic manner. However,

\footnotetext{
7 The mass and distance measurements are strongly correlated, with mass scaling roughly as $M \propto D^{1.8}$, resulting in a somewhat smaller uncertainty in the corresponding angular size (Ghez et al. 2008).
}

special care must be taken to avoid the region very close to the central object where the quasi-Kerr metric becomes unphysical (Johannsen 2013c). Our analysis properly takes this requirement into account and makes a conservative assessment of potential deviations from the Kerr metric induced by such a quadrupole moment.

Already, a variety of potential lensing signatures of deviations from general relativity within the context of the quasi-Kerr metric have been identified. While the shadow of a Kerr black hole is nearly circular, except for extreme black hole spins and viewing angles, ${ }^{8}$ quasi-Kerr-type quadrupolar metric deviations typically induce substantial asymmetries (Johannsen \& Psaltis 2010b). Similar asymmetries are present for compact objects within the context of a variety of alternative gravity theories (see, e.g., Bambi \& Yoshida 2010; Amarilla et al. 2010; Bambi et al. 2012; Amarilla \& Eiroa 2012; Johannsen 2013a).

We expand on these existing studies here by repeating the analysis of Broderick et al. (2011b) employing the quasi-Kerr metric. Questions of particular interest include:

1. How robust are existing estimates of black hole spin (magnitude and direction) to additional metric degrees of freedom? That is, how well is spin measured currently?

2. Which, if any, of the previously identified signatures of deviations from the Kerr metric persist once the additional astrophysical degrees of freedom are introduced?

3. What constraints can be placed on deviations from the Kerr metric with the current Event Horizon Telescope (EHT) data?

Generally, we find that the black hole spin orientation is extremely robust to quadrupolar perturbations, with the inclination and position angle limited to $\theta=65^{\circ}+21^{\circ}$ and $\xi=$ $127_{-14^{\circ}}^{\circ}$ (modulo an intrinsic $180^{\circ}$ uncertainty), respectively. Similarly, despite being strongly correlated with the quadrupolar perturbation, after marginalization over it, the spin magnitude posterior probability distribution is also quite robust, implying a dimensionless spin parameter of $a_{*}=0^{+0.7}$.

We find that the images of RIAFs around black holes with nonzero quadrupolar deviations differ significantly from those without. We confirm the general trend of deformed silhouettes in these images. However, we also identify additional morphological signatures due to alterations in the dynamical structure of the accretion flow. These are not unrelated: at the wavelengths accessible to mm-VLBI the detailed structure of the black hole shadow (and so-called photon ring) is partially obscured by the underlying accretion flow. Despite this, it remains a key feature in distinguishing the underlying spacetime metric.

As expected, the current limits on quadrupolar deviations from mm-VLBI observations are currently quite weak given the sparse data coverage of the $u-v$ plane. Nevertheless, features are identified in the images and mm-VLBI observables that will be accessible to future observations.

In the remaining subsections we introduce the EHT, an existing mm-VLBI telescope, and tests of the no-hair theorem using phenomenological Kerr-like spacetimes. In Section 2 we describe the quasi-Kerr metric, highlighting its features and limitations. Section 3 describes how the accretion flow is modeled, imaged, and VLBI observables are extracted. Fitting and parameter estimation procedures are discussed in Section 4,

\footnotetext{
8 Moderate deviations from a circular shadow appear only for dimensionless spin parameters of $\left|a_{*}\right| \gtrsim 0.9$ oriented at large oblique inclinations.
} 
with results collected in Section 5. Finally, conclusions are formulated in Section 6. Unless otherwise noted, we adopt units in which $G=c=1$, where $G$ and $c$ are Newton's constant and the speed of light, respectively.

\subsection{The Event Horizon Telescope}

The EHT is an evolving collection of millimeter and submillimeter observatories equipped with VLBI instrumentation for the purpose of producing horizon-resolving studies of supermassive black holes at $1.3 \mathrm{~mm}(230 \mathrm{GHz})$ and $0.87 \mathrm{~mm}(345 \mathrm{GHz})$; see Fish et al. (2013) for a recent summary of the instrument. Demonstration experiments were performed in 2007 and 2009, in which the black hole at the center of the Milky Way, Sgr A*, was observed (Doeleman et al. 2008; Fish et al. 2011). These provided the first conclusive evidence for the presence of subhorizon-scale structure in the emission region of Sgr A*. These have been followed by horizon-resolving observations of M87, a known radio jet source (Doeleman et al. 2012).

To date, observations by the EHT have been successfully performed with only three stations: the James Clerk Maxwell Telescope (JCMT) and Sub-Millimeter Array (SMA) on Mauna Kea in Hawaii, the Combined Array for Research in Millimeterwave Astronomy (CARMA) in Cedar Flat, California, and the Arizona Radio Observatory Sub-Millimeter Telescope (SMT) on Mount Graham, Arizona. The longest baseline is roughly $4.6 \times 10^{3} \mathrm{~km}$ and extends mostly east-west between Hawaii and the SMT. Because of the limited number of stations, it is not currently possible to generate images, requiring that physical analyses be performed using the visibilities directly.

Of the two sources observed by the EHT, Sgr A* has been observed most often and has been the subject of the vast majority of the published analyses to date. Thus, here we will focus our attention on Sgr A* as well. The currently available data for Sgr A* are composed of observations during two days in 2007 (April 11 and 12, Doeleman et al. 2008) and three days in 2009 (April 5-7, Fish et al. 2011). Within both observing periods Sgr A* was in a quiescent state (i.e., did not exhibit any flaring). Nevertheless, substantial flux variations were observed, both between 2007 and 2009 and on day timescales within 2009. Thus, the data are conveniently separated into four observational epochs consisting of all days in 2007 and each individual day in 2009. We label the former simply as 2007 and the latter by their day, i.e., 2009.95, 2009.96, and 2009.97.

In addition to visibility magnitudes, Fish et al. (2011) reported the first detection of a closure phase on the Hawaii-CARMA-SMT triangle of $0^{\circ} \pm 40^{\circ}$. Despite the large error bars, this is quite constraining due to the sensitivity of closure phases to the underlying source structure (Broderick et al. 2011a). Thus, we include this closure phase measurement in our parameter estimation.

The EHT is expected to grow substantially in the future, both in sensitivity and in the number of stations. Additional sites already in the process of being included are the Atacama Large Millimeter Array (ALMA) in Chile, the Large Millimeter Telescope (LMT) in Mexico, and the South Pole Telescope (SPT). All correspond to long north-south baselines that critically complement the already-existing long east-west baselines. Furthermore, the exquisite sensitivity of ALMA promises to enable the inclusion of a number of smaller sites, as well as dramatic enhancements in the signal-to-noise ratio of existing sites. Thus, an assessment of the current and future ability of the EHT to constrain the properties of the spacetime is especially timely. Here we focus on the current capability, outlining the procedure and discussing the implications for previous analyses. We will treat the future capability elsewhere.

\subsection{Parametric Tests of the No-hair Theorem}

According to the no-hair theorem, a stationary black hole in general relativity is uniquely characterized by only two parameters, ${ }^{9}$ its mass $M$ and its spin $J$, and is described by the Kerr metric (see, e.g., Heusler 1996). Dynamical features, associated, e.g., with black hole mergers, are expected to damp on timescales comparable to the light-crossing time of the black hole, which even for the most massive cases is on the order of days. Hence, it is generally anticipated that all astrophysical black holes are well described by the Kerr metric.

Several tests of the no-hair theorem have been proposed to date. These may be conveniently grouped into tests that probe the spacetime at locations far from and near the horizon. Examples of the former include high-precision studies of stellar orbital dynamics around Sgr A* (Will 2008; Merritt et al. 2010) and timing measurements of pulsar-black hole binaries (Wex \& Kopeikin 1999; Liu et al. 2012), which have taken on new importance following the discovery of a pulsar in the Galactic center (Kennea et al. 2013). Proposed tests of the strong-field properties of astrophysical black holes are based either on the gravitational waves generated by stellar-mass compact objects falling into supermassive black holes in extreme mass-ratio inspirals (EMRIs; for a review, see Gair et al. 2013) or on the electromagnetic radiation emitted from black hole accretion disks (Johannsen \& Psaltis 2010a). In order to properly model the expected signals of these tests, both approaches generally modify the Kerr metric using a parametrically deformed Kerrlike spacetime, which contains one or more additional free parameters and which reduces to the Kerr metric if all deviations vanish (e.g., Manko \& Novikov 1992; Collins \& Hughes 2004; Glampedakis \& Babak 2006; Vigeland \& Hughes 2010; Johannsen \& Psaltis 2011a; Vigeland et al. 2011; Johannsen 2013b). A measurement of these deviations can then provide a parameterized test of the applicability of the Kerr metric to astrophysical black holes (Ryan 1995).

Here we employ a multipole expansion of the Kerr metric. In analogy to Newtonian gravity, where the potential $\Phi$ of a given mass distribution can be expanded in a series of spherical harmonics whose coefficients are the multipole moments of the source mass, in general relativity a multipole expansion can be constructed that describes the mass and matter current distribution of the spacetime (see, e.g., Thorne 1980). The nohair theorem immediately implies that all higher-order multipole moments of a Kerr black hole are not independent, but rather can be expressed in terms of the mass and spin. This relationship can be expressed particularly compactly via

$$
M_{l}+i S_{l}=M(i a)^{l}
$$

where $M_{l}$ and $S_{l}$ are the mass and matter current multipole moment and $a \equiv J / M$, where $J$ is the black hole angular momentum (Geroch 1970; Hansen 1974). In this expansion, mass and angular momentum may be identified with the first two multipoles of the spacetime, i.e., $M_{0}=M$ and $S_{1}=J$. Of particular importance here is the next order, the quadrupole mass moment:

$$
Q_{\mathrm{K}} \equiv M_{2}=-M a^{2} .
$$

\footnotetext{
9 In principle, stationary black holes can also have an electric charge. However, in astrophysical settings, in which ions are available in copious quantities, it is expected that gravitationally relevant charges will neutralize rapidly.
} 
One of the simplest ways to introduce a deviation from the Kerr metric is to construct a quasi-Kerr metric (Glampedakis \& Babak 2006) as the underlying spacetime, for which the quadrupole may be set independently of the spin, having the form

$$
Q_{\mathrm{QK}}=-M\left(a^{2}+\epsilon M^{2}\right),
$$

where $\epsilon$ is a dimensionless parameter that measures potential deviations from the Kerr metric. When $\epsilon=0$, the quasi-Kerr metric reduces to the Kerr metric, and Equation (2) is trivially recovered.

Modifying the spacetime of a black hole in this manner leads to important changes of its properties that may be used to empirically test the no-hair theorem (Johannsen \& Psaltis 2010a). Most important for our purposes here are the modifications of the null geodesic structure of the spacetime. This results in characteristic distortions of the black hole shadow, the lensed image of the black hole horizon cast against a putative surrounding accretion flow, and the surrounding photon ring, which corresponds to the projection along null geodesics of the orbits of photons that propagate around the black hole many times before they are observed. While this ring is exactly circular for a Schwarzschild black hole and nearly circular for a Kerr black hole with spin values $a / M \lesssim 0.9$, the shape of this ring becomes asymmetric if the no-hair theorem is violated (Johannsen \& Psaltis 2010b). It is the prospect of detecting these signatures that we are assessing here.

For convenience, we also define a dimensionless spin parameter $a_{*} \equiv a / M$, often referred to simply as "spin" in the literature, and the gravitational radius $r_{\mathrm{g}} \equiv G M / c^{2}$, in terms of which the Schwarzschild radius is simply $2 r_{\mathrm{g}}$.

\section{THE QUASI-KERR METRIC}

In this section, we describe the quasi-Kerr metric (Glampedakis \& Babak 2006) and summarize some of its properties, which were analyzed in detail in Johannsen \& Psaltis (2010a) and Johannsen (2013c). The quasi-Kerr metric (Glampedakis \& Babak 2006) derives from the Hartle-Thorne metric (Hartle 1967; Hartle \& Thorne 1968), originally designed to describe the exterior spacetimes of slowly rotating neutron stars. Glampedakis \& Babak (2006) considered this metric to be a small perturbation of the Kerr metric and used it to construct approximate waveforms for EMRIs around a non-Kerr compact object. In this context, the quasi-Kerr spacetime becomes accessible to particles at very small radii that otherwise lie below the neutron star surface and is formally applicable to black holes with small spins. This metric, then, generally harbors a naked singularity ${ }^{10}$ (Johannsen 2013c). Subsequently, Johannsen \& Psaltis (2010a) appropriated this metric as a framework for tests of the no-hair theorem based on the electromagnetic radiation emitted from the accretion flows around black holes.

Treating the quasi-Kerr metric as a small perturbation to the Kerr metric, however, is not entirely possible within the context of ray-tracing computations because the geodesic equations are nonlinear and the perturbative nature of the metric cannot be consistently enforced. Instead, we treat the quasi-Kerr metric as an "exact" metric with a deformed quadrupole moment and with the same higher-order multipole moments as the Kerr metric, permitting the study of the effects of a deviation from the Kerr metric at the quadrupole order (see the discussion

10 This may be explicitly verified by a careful examination of the Kretschmann scalar, $K \equiv R^{\alpha \beta \gamma \delta} R_{\alpha \beta \gamma \delta}$, where $R^{\alpha \beta \gamma \delta}$ is the Riemann curvature tensor. in Johannsen 2013c). Therefore, no longer constrained by the need to maintain a perturbation expansion and given that such deviations are largely unconstrained empirically, we do not limit our analysis to small values of spin either, considering values up to $a_{*}=0.9$. However, precautions must be taken to avoid any contact of photons and particles in the accretion flow with the naked singularity at small radii, which restricts the range of quadrupolar deviations that we may consider such that the quasi-Kerr metric remains a valid framework for our analysis (see Johannsen 2013c). We describe these restrictions in detail below.

In Boyer-Lindquist coordinates $(t, r, \theta, \phi)$, the quasi-Kerr metric can be described by the line element (Glampedakis \& Babak 2006)

$$
d s^{2}=g_{t t} d t^{2}+2 g_{t \phi} d t d \phi+g_{r r} d r^{2}+g_{\theta \theta} d \theta^{2}+g_{\phi \phi} d \phi^{2},
$$

where

$$
\begin{aligned}
& g_{t t}=-\left(1-\frac{2 M r}{\Sigma}\right)+\frac{5 \epsilon(1+3 \cos 2 \theta)}{32 M^{2} r^{2}} \\
& \times\left[2 M\left(3 r^{3}-9 M r^{2}+4 M^{2} r+2 M^{3}\right)\right. \\
& \left.-3 r^{2}(r-2 M)^{2} \ln \left(\frac{r}{r-2 M}\right)\right] \text {, } \\
& g_{t \phi}=-\frac{2 M a r \sin ^{2} \theta}{\Sigma}, \\
& g_{r r}=\frac{\Sigma}{\Delta}-\frac{5 \epsilon\left(1-3 \cos ^{2} \theta\right)}{16 M^{2}(r-2 M)^{2}} \\
& \times\left[2 M(r-M)\left(3 r^{2}-6 M r-2 M^{2}\right)\right. \\
& \left.-3 r^{2}(r-2 M)^{2} \ln \left(\frac{r}{r-2 M}\right)\right] \text {, } \\
& g_{\theta \theta}=\Sigma-\frac{5 \epsilon r(1+3 \cos 2 \theta)}{32 M^{2}}\left[-2 M\left(3 r^{2}+3 M r-2 M^{2}\right)\right. \\
& \left.+3 r\left(r^{2}-2 M^{2}\right) \ln \left(\frac{r}{r-2 M}\right)\right], \\
& g_{\phi \phi}=\left[r^{2}+a^{2}+\frac{2 M a^{2} r \sin ^{2} \theta}{\Sigma}-\frac{5 \epsilon r(1+3 \cos 2 \theta)}{32 M^{2}}\right. \\
& \times\left[-2 M\left(3 r^{2}+3 M r-2 M^{2}\right)\right. \\
& \left.\left.+3 r\left(r^{2}-2 M^{2}\right) \ln \left(\frac{r}{r-2 M}\right)\right]\right] \sin ^{2} \theta,
\end{aligned}
$$

in which

$$
\Delta \equiv r^{2}-2 M r+a^{2} \text { and } \Sigma \equiv r^{2}+a^{2} \cos ^{2} \theta .
$$

For nonzero values of the parameter $\epsilon$, the properties of the quasi-Kerr spacetime are altered in several key aspects (Johannsen \& Psaltis 2010a): (1) the locations of the circular photon orbit and the innermost stable circular orbit (ISCO) are shifted, (2) trajectories of photons near the compact object are gravitationally lensed to a stronger or lesser degree, and (3) observed photon redshifts are altered, both potentially increased or decreased. All of these features lead to a modified image of any putative accretion flow.

Very close to the naked singularity, the quasi-Kerr metric contains regions where the metric signature is no longer Lorentzian 
and where closed timelike curves exist (Johannsen 2013c). Since all of these pathologies are unphysical, we introduce a cutoff radius $r_{\text {cutoff }}$ outside of which the metric is well behaved. Any particle that enters the region where $r<r_{\text {cutoff }}$ is considered captured by the black hole. Thus, the cutoff radius acts as an artificial horizon, which, for the purposes of this paper, allows us to effectively treat the central object as a black hole and ensures that all unphysical regions are shielded from distant observers (Johannsen \& Psaltis 2010a).

Here we choose to place the cutoff at a fixed radius $r_{\text {cutoff }}=$ $3 r_{\mathrm{g}}$, the radius of the circular photon orbit of a Schwarzschild black hole, which facilitates the comparison of images with different values of the spin and the deviation parameter. With this definition of the cutoff radius we ignore the emission and propagation of photons inside this region. This implies that for some values of $a$ and $\epsilon$ the photon orbit lies within $r_{\text {cutoff }}$, and therefore, the innermost portion of the image has possibly been neglected. As might be expected, this results in a marginally larger black hole shadow. More importantly, the neglected part of the image includes the photon ring, the shape of which directly imprints the structure of the spacetime (Johannsen \& Psaltis 2010b). Thus, by imposing a cutoff radius we necessarily ignore features that are particularly sensitive to quadrupolar deviations for $\epsilon \lesssim 0$. Extending $r_{\text {cutoff }}$ inside of $3 r_{\mathrm{g}}$ would generally result in more extreme deviations and thus stronger limits on $\epsilon$.

Furthermore, to avoid possible artifacts associated with our choice of $r_{\text {cutoff }}$, we restrict our attention to parameter choices for which the ISCO is larger than $4 r_{\mathrm{g}}$. Since the location of the ISCO is a function of both the spin and the deviation parameter $\epsilon$, this necessarily limits the parameter space we may consider, with the minimum permissible value of the parameter $\epsilon$ being a function of the spin. At vanishing spin, permissible metrics can be found for $\epsilon \gtrsim-0.8$, below which the ISCO lies within $4 r_{\mathrm{g}}$. Thus, for concreteness, we restrict ourselves to $-0.8 \leqslant \epsilon \leqslant 1$. Since high spin has already been found to be highly disfavored, we also limit ourselves to $0 \leqslant a_{*} \leqslant 0.9$. However, the condition on the ISCO implies that not all $(a, \epsilon)$ within these ranges are permissible: higher spin values generally result in smaller ISCOs and therefore more stringent limits on the deviation parameter (see Figure 3).

In practice, $r_{\text {cutoff }}$ represents a choice for how solutions to a modified theory of strong gravity will alter the Kerr metric near the horizon. Without the introduction of a cutoff radius, images of accretion flows would reveal what we consider to be artificial features that arise from the pathological regions that lie inside of this radius. In particular, for positive values of the parameter $\epsilon$, images show two bright spots, which are located along the spin axis of the black hole inside of the domain of the shadow. For negative values of the parameter $\epsilon$, images contain a ringlike band that stretches across the center of the image. These features arise due to regions in which $g_{r r}<0$; that is, the compact object has effectively become repulsive. By excluding these regions from our analysis, we necessarily restrict ourselves to observable signatures arising from regions in which the quasiKerr metric is well defined. Hence, in this sense, we are making the most conservative comparison possible with this context.

The shadow cast by the black hole is shown in Figure 1 for a nonrotating black hole at various values of $\epsilon$. The qualitative impact is precisely that expected by Johannsen \& Psaltis (2010a): more prolate spacetimes $(\epsilon<0)$ produce extended silhouettes along the coordinate axis, while more oblate spacetimes $(\epsilon>0)$ produce extended silhouettes in the equatorial plane. Even for order-unity quadrupolar deviations, the distortions are relatively small in comparison to the unperturbed shadow, increasing the semimajor axis of the shadow by roughly $7 \%$ at $|\epsilon| \simeq 0.8$. For Sgr A*, this implies variations in the image structure on scales of order $4 \mu$ as or smaller. Nevertheless, we will see that this degree of image variations is readily distinguishable even with the existing mm-VLBI data.

\section{VISIBILITY MODELING}

Because of the limited $u-v$ coverage of the early EHT observations of $\mathrm{Sgr} \mathrm{A}^{*}$, it is not currently possible to construct high-fidelity image reconstructions from the reported visibilities. Nor is it clear that analyzing images is intrinsically more desirable. Thus, it is necessary to construct visibility models based on physical models of the spacetime and the surrounding emission regions. In the previous section, we described the spacetime, from which it is straightforward to compute photon trajectories (i.e., null geodesics). Here we describe the model we use for the emission region, which is based on the RIAF models employed in Broderick et al. (2011b). Because this has been described at length there (and references therein), here we provide only a cursory summary of the model details, referring the interested reader to those earlier studies for the particular justifications.

Thus, we expect that the limits on $\epsilon$ obtained here are pessimistic in practice. Note that for this reason the parameter estimates from the existing EHT data at $\epsilon=0$ are slightly different from those found in Broderick et al. (2011b).

\subsection{Accretion Flow Modeling}

Sgr $A^{*}$ transitions from an inverted, presumably optically thick spectrum to an optically thin spectrum near millimeter wavelengths. This implies that near $1.3 \mathrm{~mm} \mathrm{Sgr} \mathrm{A*} \mathrm{is} \mathrm{only}$ becoming optically thin, and thus absorption in the surrounding medium is likely to be important. This transition does not occur isotropically, happening at longer wavelengths for gas that is receding and at shorter wavelengths for gas that is approaching. Therefore, proper modeling of the structure and relativistic radiative transfer is crucial to producing high-fidelity images.

Metric perturbations impact the accretion flow in a number of ways, substantially modifying its dynamics and the lensing of the emitted radiation, as well as potentially affecting its spatial structure. To directly compare with the analyses in Broderick et al. (2011b), we will ignore alterations to the flow structure (in a coordinate sense) and focus solely on the observational signatures associated with lensing and dynamics. This is motivated in part by the dominance of the latter effects observed in studies of the impact of black hole spin and suggested below (Section 5.2), where it is found that spacetime modifications are manifested in the resulting images primarily via the location of the ISCO (albeit within the constrained model class described here). It is also a result of expedience; a full exploration of self-consistent disk modeling in modified spacetimes is beyond the scope of this paper.

For concreteness, as in Broderick et al. (2011b), we follow Yuan et al. (2003) and employ a model in which the accretion flow has a population of thermal electrons with density and temperature

$$
n_{e, \text { th }}=n_{e, \text { th }}^{0}\left(\frac{r}{2 r_{\mathrm{g}}}\right)^{-1.1} e^{-z^{2} / 2 \rho^{2}}
$$



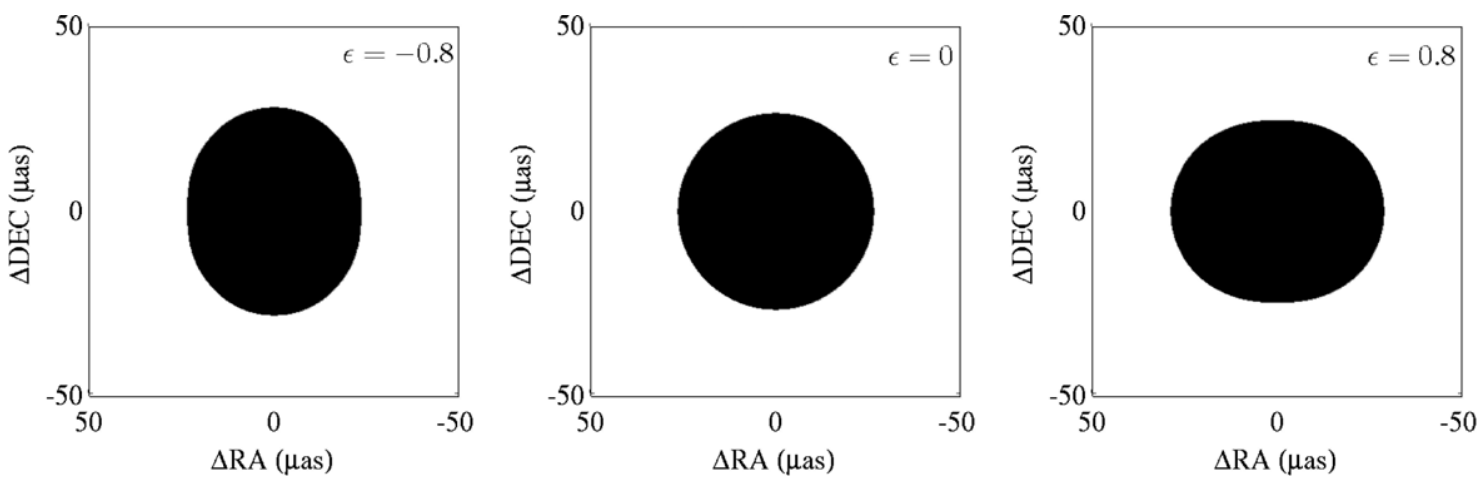

Figure 1. Example black hole silhouettes for nonrotating black holes with illustrative values of the quadrupolar parameter and applying a cutoff radius of $r_{\text {cutoff }}=3 r_{\mathrm{g}}$, as described in the text. In all cases $\theta=90^{\circ}$, and the coordinate axis is parallel to the vertical direction. For negative values of the deviation parameter $\epsilon$ (left panel), the shadow has a more prolate shape than the shadow of a Kerr black hole with the same spin (central panel), while for positive values of the deviation parameter (right panel), the shadow has a more oblate shape.

and

$$
T_{e}=n_{e}^{0}\left(\frac{r}{2 r_{\mathrm{g}}}\right)^{-0.84},
$$

respectively, and a toroidal magnetic field in approximate $(\beta=10)$ equipartition with the ions (which are responsible for the majority of the pressure), i.e.,

$$
\frac{B^{2}}{8 \pi}=\beta^{-1} n_{e, \mathrm{th}} \frac{m_{p} c^{2} r_{\mathrm{g}}}{6 r} .
$$

In Equation (7), $\rho$ is the cylindrical radius and $z$ is the vertical coordinate.

Beyond the ISCO the accretion flow orbits with the angular velocity of the equatorial stable circular orbit with the same cylindrical radius, i.e., the relativistic analogs of Keplerian orbits. ${ }^{11}$ Inside of the ISCO we assume that the gas is plunging on ballistic trajectories. In principle, the plunging gas can still radiate, although in practice it contributes little to the overall emission due to the large radial velocities it develops.

In the case of the thermal quantities the radial structure was taken from Yuan et al. (2003), and the vertical structure was determined by assuming that the disk height is comparable to $\rho$. Note that all of the models we employ necessarily have the spin aligned with the orbital angular momentum of the accretion flow. For the regions that dominate the millimeter emission it is unclear if this is a good assumption for RIAF models generally and Sgr A* specifically. Assessing the restriction this implies will be considered in future work.

Thermal electrons alone are incapable of reproducing the nearly flat spectrum of Sgr A* below $43 \mathrm{GHz}$. Thus, it is necessary to also include a nonthermal component. As with the thermal components, we adopt a self-similar model for a population of nonthermal electrons,

$$
n_{e, \text { nth }}=n_{e, \text { nth }}^{0}\left(\frac{r}{2 r_{\mathrm{g}}}\right)^{-2.02} e^{-z^{2} / 2 \rho^{2}}
$$

with a power-law distribution corresponding to a spectral index of 1.25 and cut off below Lorentz factors of $10^{2}$ (consistent with Yuan et al. 2003). The radial power-law index was chosen to reproduce the low-frequency spectrum of $\mathrm{Sgr} \mathrm{A}^{*}$ and is insensitive to the black hole properties due to the distant origin of the long-wavelength emission.

\footnotetext{
11 With the exception that when the quasi-Kerr metric is employed, this is
} identical to the orbital velocities considered in Broderick et al. (2011b).
The primary emission mechanism at the wavelengths of interest is synchrotron radiation, arising from both the thermal and nonthermal electrons. We model the emission from the thermal electrons using the emissivity described in Yuan et al. (2003), appropriately altered to account for relativistic effects (see, e.g., Broderick \& Blandford 2004). Since we perform polarized radiative transfer via the entire complement of Stokes parameters, we employ the polarization fraction for thermal synchrotron as derived in Petrosian \& McTiernan (1983). In doing so, we have implicitly assumed that the emission due to thermal electrons is isotropic, which, although generally not the case, is unlikely to change our results significantly. For the nonthermal electrons, we follow Jones \& O'Dell (1977) for a power-law electron distribution, with an additional spectral break associated with the minimum electron Lorentz factor. For both emission components the absorption coefficients are determined directly via Kirchhoff's law. Images are then produced using the fully relativistic ray-tracing and radiative transfer schemes described in Broderick \& Loeb (2006a, 2006b) and Broderick (2006), into which the quasi-Kerr metric described in Section 2 is inserted.

Because Yuan et al. (2003) neglected relativistic effects and assumed spherical symmetry, their models are not directly applicable here. For these reasons, as in Broderick et al. (2011b), at each point of interest in the $a-\theta-\epsilon$ parameter space, the coefficients $\left(n_{e, \text { th }}^{0}, T_{e}^{0}, n_{e, \text { nth }}^{0}\right)$ were adjusted to fit the radio spectral energy distribution (SED) of Sgr A* (for details on the fitting procedure, see Broderick et al. 2011b). In all cases it was possible to fit the SED with extraordinary precision.

Because of its time-consuming nature, the spectral fitting procedure was performed at a subset of points in the $a-\theta-\epsilon$ parameter space. Explicitly, for values of the spin and the deviation parameter for which the ISCO exceeded the $4 r_{\mathrm{g}}$ limit, fits were performed at values of $\epsilon$ ranging from -0.8 to 1 in steps of $0.1, a$ from 0 to 0.9 in steps of 0.1 , and $\theta$ from $0^{\circ}$ to $90^{\circ}$ in steps of $10^{\circ}$. Values for the fitted coefficients $\left(n_{e, \mathrm{th}}^{0}, T_{e}^{0}, n_{e, \mathrm{nth}}^{0}\right)$ were then obtained at arbitrary points via high-order polynomial interpolation.

During the $1.3 \mathrm{~mm}$ VLBI observations, Sgr A*'s flux varied by roughly $30 \%$. We model this as a variable accretion rate, allowing the electron density normalization to vary, corresponding to a modulation of the accretion rate. In practice, we reduced the electron density normalization by an amount sufficient to produce a total flux of $2.5 \mathrm{Jy}$ and then multiplied the resulting images by a correction factor during the EHT data analysis. Because the source is not uniformly optically thin, this 

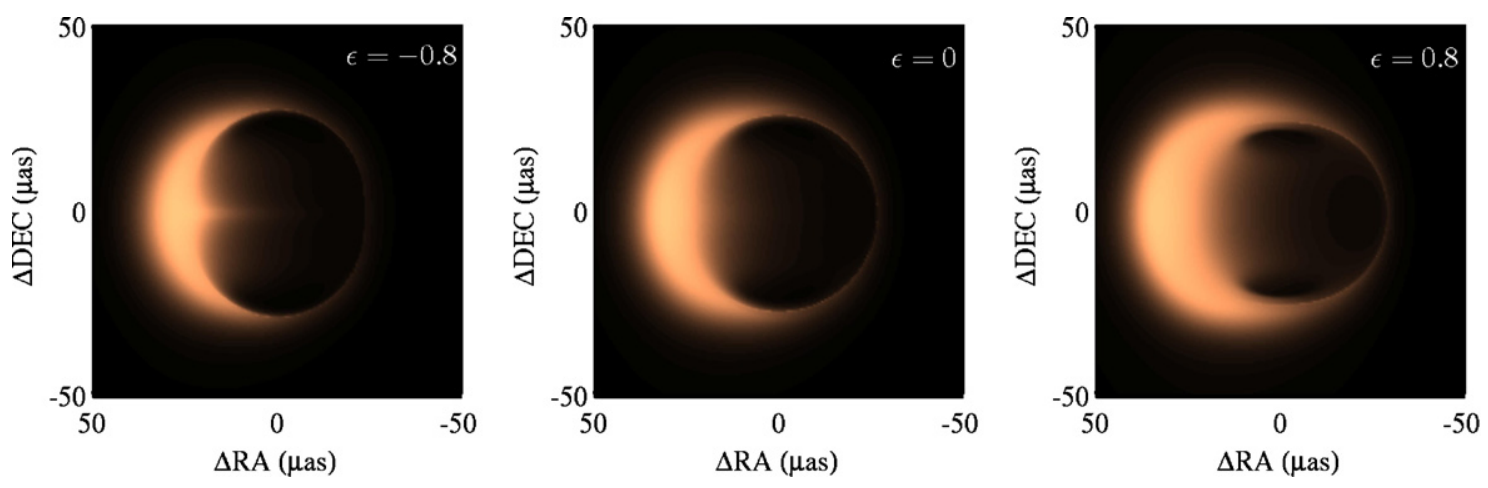

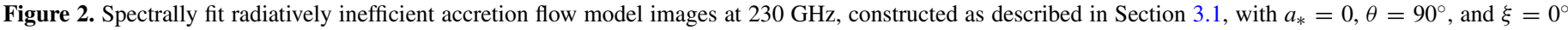

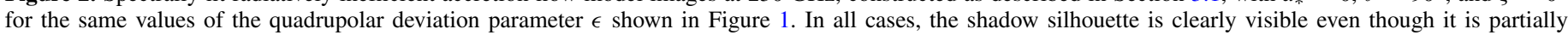

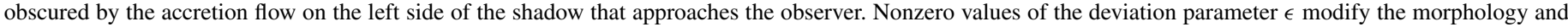

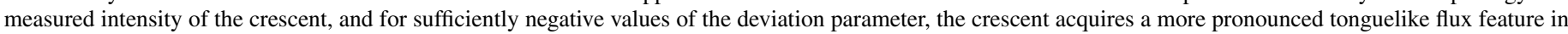
the equatorial plane of the black hole.

(A color version of this figure is available in the online journal.)

is not strictly correct, although the associated error in the image structure is small.

Finally, arbitrary position angles $\xi$ are modeled simply by rotating the images on the sky. For this purpose we define $\xi$ as the position angle (east of north) of the projected spin vector.

A collection of example images constructed via the above procedure is presented in Figure 2, corresponding to the same spacetimes used to construct the silhouettes in Figure 1. In all cases they exhibit the crescent morphology characteristic of thick accretion flows, arising because of the relativistic orbital motions: both Doppler beaming and boosting result in an enhancement and reduction of the surface brightness and optical depth on the approaching (left) and receding (right) sides of the accretion flow, respectively.

In all images the silhouette of the black hole is clearly visible, varying qualitatively, as anticipated by Figure 1. The detailed structure of the shadow, however, is partially obscured by the optically thick crescent on the approaching side of the accretion flow. Thus, as expected, the additional degrees of freedom in the accretion flow do slightly moderate the impact of the spacetime on the apparent shape of the shadow itself (see Figures 2 and 1).

However, more importantly, additional features not present in the silhouettes alone appear. These include the equatorial tonguelike flux feature in the left panel of Figure 2, which is primarily caused by the enhanced Doppler boosting of the accretion flow particles near the ISCO, and the variation in the crescent width due to modifications in the orbital dynamics of test particles. Thus, the images contain a substantial amount of information about the spacetime, both directly via the shadow shape and the null geodesic structure and indirectly via the impact of the spacetime on the matter dynamics and hence the emission and subsequent radiative transfer through the accretion flow.

\subsection{Computing Visibility Magnitudes and Closure Phases}

Given a trial image intensity distribution $I(\alpha, \beta)$, where $\alpha$ and $\beta$ are angular coordinates, we may compute the visibilities in the standard fashion:

$$
V(u, v)=\iint d \alpha d \beta e^{-2 \pi i(\alpha u+\beta v) / \lambda} I(\alpha, \beta) .
$$

To compute the visibility magnitudes, these must then be modified to account for the blurring effects due to electron scattering resulting from propagation through the Galactic plane.

The effect of interstellar electron scattering in the direction of Sgr $A^{*}$ has been carefully characterized empirically by a number of authors. This has been found to be consistent with convolving the source with an asymmetric Gaussian, with the major axis nearly aligned with east-west and a $\lambda^{2}$ wavelength dependence. We employ the model from Bower et al. (2006), which has the major axis oriented $78^{\circ}$ east of north, with the associated FWHM for the major and minor axes given by

$$
\begin{aligned}
& \mathrm{FWHM}_{M}^{\mathrm{ES}}=1.309\left(\frac{\lambda}{1 \mathrm{~cm}}\right)^{2} \mathrm{mas}, \\
& \mathrm{FWHM}_{m}^{\mathrm{ES}}=0.64\left(\frac{\lambda}{1 \mathrm{~cm}}\right)^{2} \mathrm{mas},
\end{aligned}
$$

respectively. In practice, the interstellar electron scattering convolution was effected in the $u-v$ plane, where the convolution reduces to a multiplication.

Finally, closure phases are obtained for a given triplet of VLBI stations via their definition: the closure phase for a given triplet of observatories is then given by

$$
\Phi_{i j k}=\arg \left[V\left(u_{i j}, v_{i j}\right)\right]+\arg \left[V\left(u_{j k}, v_{j k}\right)\right]+\arg \left[V\left(u_{k i}, v_{k i}\right)\right] .
$$

In practice, where this required some interpolation, it was performed on the real and imaginary components of $V$, which were then used to reconstruct the phases.

\section{VISIBILITY FITTING AND PARAMETER ESTIMATION}

Comparing the models described in Section 3 with the existing EHT observations presents both practical and conceptual difficulties. The construction of large libraries of spectrally fit models is a computationally expensive exercise, which becomes exponentially harder as additional parameters are considered. Here we describe the scheme by which our model library was constructed and summarize the statistical measures of the fits employed.

\subsection{Model Library Construction and Comparison Efficiency}

The analysis of the EHT data within the context of RIAF models that assume general relativity described in Broderick 
et al. (2011b) already taxed the substantial computational resources available. This was a result of both the process of spectral fitting and the large-scale visibility generation and comparisons. Thus, the addition of a quadrupole parameter required the development of new analysis techniques. These techniques came in two forms: increasing the speed with which a model at a given point in the parameter space can be compared to the data and more efficiently choosing regions within the parameter space to search for satisfactory models.

\subsubsection{Improved Model Comparison Efficiency}

In practice, comparing a model to the EHT data is performed via the following steps:

1. Compute an image of the model with the given parameters at $1.3 \mathrm{~mm}$ with an appropriate flux normalization.

2. From the image, compute the complex visibilities via the Fast Fourier Transform algorithm, up to an uncertain epochdependent flux normalization.

3. Obtain the complex visibilities at the points in the $u$ - $v$ plane at which the visibility magnitudes were measured. Note that in practice this is done for a large number of position angles at one time, corresponding to a rotation of the image.

4. From the complex visibilities compute the magnitudes at the relevant $u-v$ positions and closure phases on the relevant triangles.

5. Construct a likelihood by direct comparison to the data.

6. Marginalize or maximize over the epoch-dependent flux normalizations.

The first step requires large-scale computing to complete in a reasonable amount of time, typically weeks. Optimizing the first is the subject of the following subsection. The remaining steps are performed on a workstation, typically taking days. This duration is dominated by the time associated with constructing the likelihoods and marginalizing over the flux normalizations. The reason is the presence of a visibility magnitude upper limit in 2007, which requires computing an error function and, most importantly, forcing a numerical approach to the marginalization.

However, it is possible to analytically perform the marginalizations if only detections (and, more specifically, measurements with Gaussian errors) are considered (see Appendices A and B). Repeating the analysis in Broderick et al. (2011b) at a lower parameter space resolution, we have verified that in light of the detections in the 2009 epochs the 2007 CARMA-Hawaii upper limit is no longer significantly constraining. By proceeding without the upper limit the analysis is sped up by roughly an order of magnitude.

A second benefit of restricting our attention to detections is that the maximum and marginalized flux normalizations are the same. Thus, it is only necessary to compute one set of quantities both to address the posterior probability density of a given set of parameters (the marginal value) and to assess the quality of the fit via a $\chi^{2}$ and/or Bayesian analysis (the maximum value).

\subsubsection{Adaptive Parameter Space Refinement}

The Broderick et al. (2011b) analysis was performed with an image library consisting of nearly 10,000 images, and once the model library was constructed (by far the most time-consuming step), it took roughly a month to complete. Even an only moderate sampling in the new parameter, $\epsilon$, would result in an intractable problem. Because of the current data quality, it is still necessary to probe large portions of the possible parameter space, with the ultimately acceptable models constituting a strongly correlated, clearly non-Gaussian subset.

The solution we have adopted is to perform repeated lowerresolution analyses, zooming in on the high-probability region. That is, the first image sample, comprising roughly 1000 images, is analyzed to obtain a rough measure of the likelihood throughout the parameter space. All regions with likelihoods larger than $10 \%$ of the maximum value were then selected for refinement, where the resolution was doubled, resulting in another 3000 images. In regions with likelihoods greater than $50 \%$ of the new maximum, a third set of images, roughly 2500 , were produced and subsequently analyzed. Finally, a third round of refinement is performed where the new likelihoods are again above $50 \%$ of the maximum value, resulting in 9000 new images.

At each refinement, the probabilities and likelihoods are interpolated to a parameter space grid of twice the resolution, with values in the refinement regions being obtained by the new analyses. Thus, the method of probing the likelihoods produces an inhomogeneous coverage of the parameter space volume, focusing attention on high-probability regions but, nevertheless, probing the whole space at least at low resolution. At the highest resolution we have $\Delta a_{*}=0.01252, \Delta \theta=1.25^{\circ}, \Delta \xi=1^{\circ}$, and $\Delta \epsilon=0.05$. Apart from the introduction of the parameter $\epsilon$, these values are marginally coarser than, although comparable to, those employed in Broderick et al. (2011b).

\section{ESTIMATION AND INTERPRETATION OF PARAMETERS}

The primary product of the forgoing analysis is the construction of a set of likelihoods at each point in the four-dimensional black hole parameter space $(a, \theta, \xi, \epsilon)$, marginalized over the four epoch-specific flux normalizations $\bar{L}(a, \theta, \xi, \epsilon)$. From this we can construct the posterior probability distributions of the parameters in the normal way (see Appendix B). In doing so we adopt flat priors on $a$ and $\epsilon$ (although see Section 5.2) and an isotropic prior on $(\theta, \xi)$. With the likelihoods and posterior parameter probabilities, here we assess the quality of the fits, obtain estimates for the black hole parameters and uncertainties, and discuss their implications.

\subsection{Assessing Fit Quality}

Prior to parameter estimation and interpretation, we address the existence of "good" fits, i.e., high likelihood fits. Given the results of Broderick et al. (2011b), we might anticipate that the presence of good fits is guaranteed. Indeed, despite the small variation due to the choice to excise the photons passing within $3 r_{\mathrm{g}}$ and the inclusion of the closure phase measurement in the analysis, we find that this remains the case. Collected in Table 1 are the $\log$ likelihood $\left(\chi^{2}\right)$, number of model parameters $(k)$, and various measures of fit quality for the analysis in Broderick et al. (2011b), with the analysis here restricted to $\epsilon=0$ and in full. These measures include the Bayesian and Akaike information criteria as defined in Broderick et al. (2011b).

Despite the inclusion of the closure phase, the distinction between the analysis in Broderick et al. (2011b) and here when restricted to Kerr is insignificant, as expected. Allowing $\epsilon \neq 0$ improves the log likelihood only marginally at the expense of an additional parameter. Thus, as will be verified explicitly in Section 5.3, little evidence exists within the existing EHT data to support the need for a non-Kerr quadrupole. 

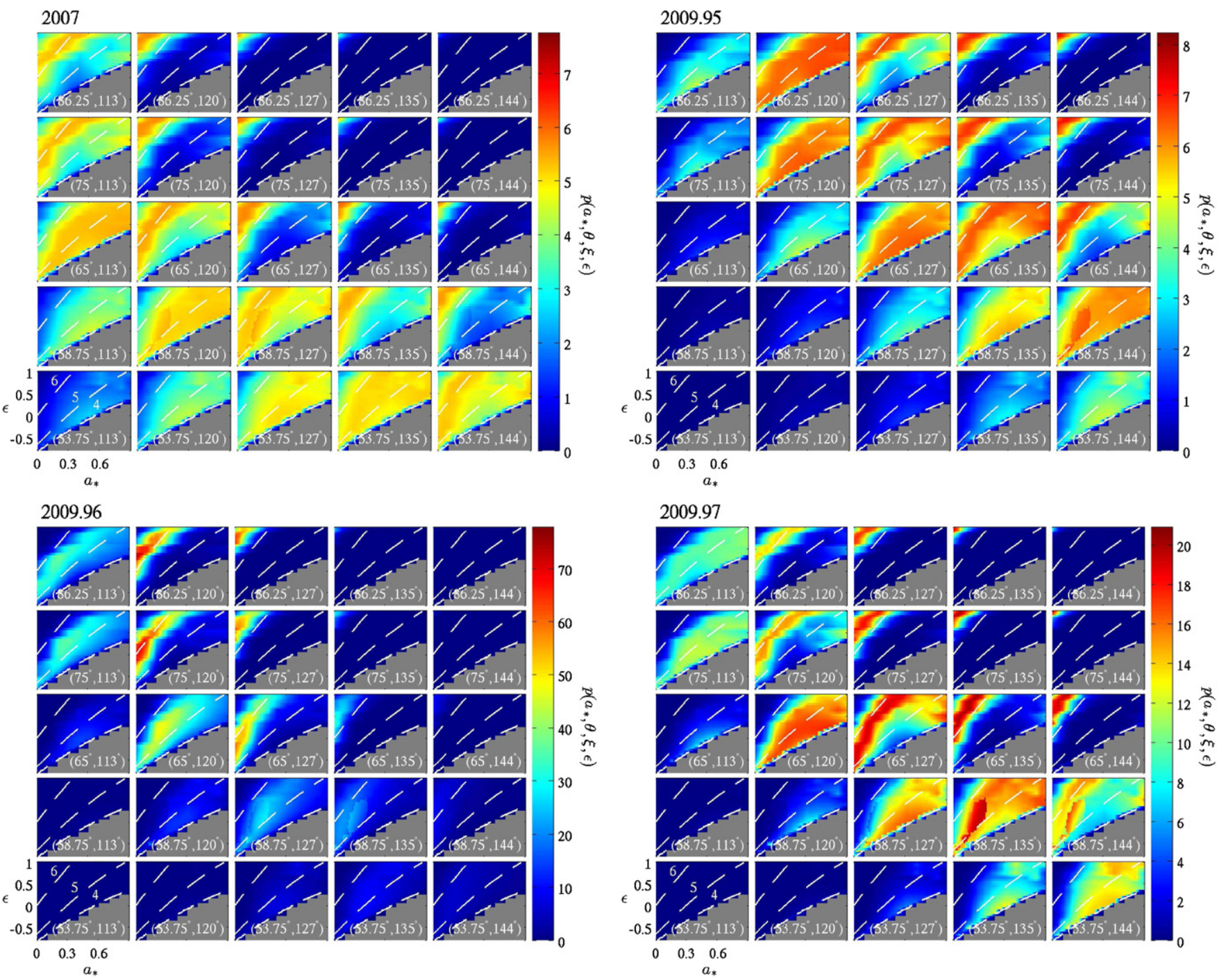

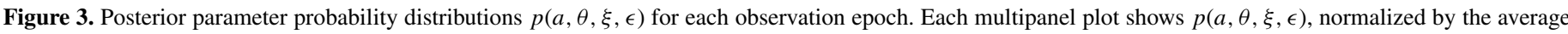

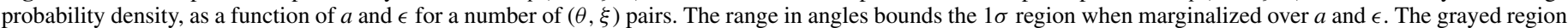

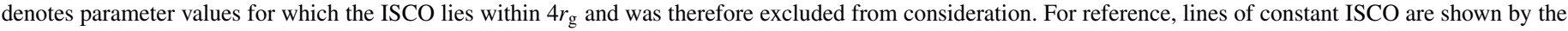
white dashed contours, corresponding to $6 r_{\mathrm{g}}, 5 r_{\mathrm{g}}$, and $4 r_{\mathrm{g}}$ (top to bottom).

(A color version of this figure is available in the online journal.)

Table 1

Model Fitting Results Summary

\begin{tabular}{lcccccc}
\hline \hline Model & $k^{\mathrm{a}}$ & $\chi^{2}$ & dof $^{\mathrm{b}}$ & $\chi^{2} /$ dof & BIC $^{\mathrm{c}}$ & AIC $^{\mathrm{d}}$ \\
\hline Broderick et al. (2011b) & 7 & 53.09 & 64 & 0.830 & 82.9 & 68.9 \\
$\epsilon=0$ & 7 & 51.79 & 65 & 0.797 & 81.7 & 67.5 \\
All $\epsilon$ & 8 & 51.74 & 64 & 0.809 & 86.0 & 70.0 \\
\hline
\end{tabular}

Notes.

a Number of model parameters, consisting of epoch flux normalizations and assumed black hole parameters.

b Associated degrees of freedom; the data used here include the closure phase reported in Fish et al. (2011) and thus include one more point than used in Broderick et al. (2011b).

c Bayesian information criterion; for a full definition, see Broderick et al. (2011b).

d Akaike information criterion; for a full definition, see Broderick et al. (2011b).

Since the black hole parameters are expected to remain fixed over the current duration of the EHT observations, we also assess the consistency of the fits across epochs. The epoch- specific posterior probability densities are shown in Figure 3 (note the differing color scales for each epoch). In these plots artifacts of the adaptive refinement scheme are clearly visible, although they primarily affect regions with probability densities below $50 \%$ of the maximum in each plot.

While at low probability density the epochs exhibit significant deviations from each other, the epochs are consistent where the probability densities are highest. This is most clearly seen by comparing the remaining epochs to 2009.96 (and, to a lesser degree, to 2009.97). All other epochs have substantial posterior probability densities in regions that are highly probable in 2009.96. Thus, a single spacetime structure is consistent with the entire set of EHT observations.

The aggregate posterior probability densities are shown in Figure 4. Many of the features exhibited on the individual epoch analyses (especially 2009.96) persist. The highest probability densities form a narrow tongue, aligned most prominently with lines of constant ISCO (shown by the white dashed lines). While the particular ISCO value varies with spin orientation, for the orientation containing the highest probability density this is at 

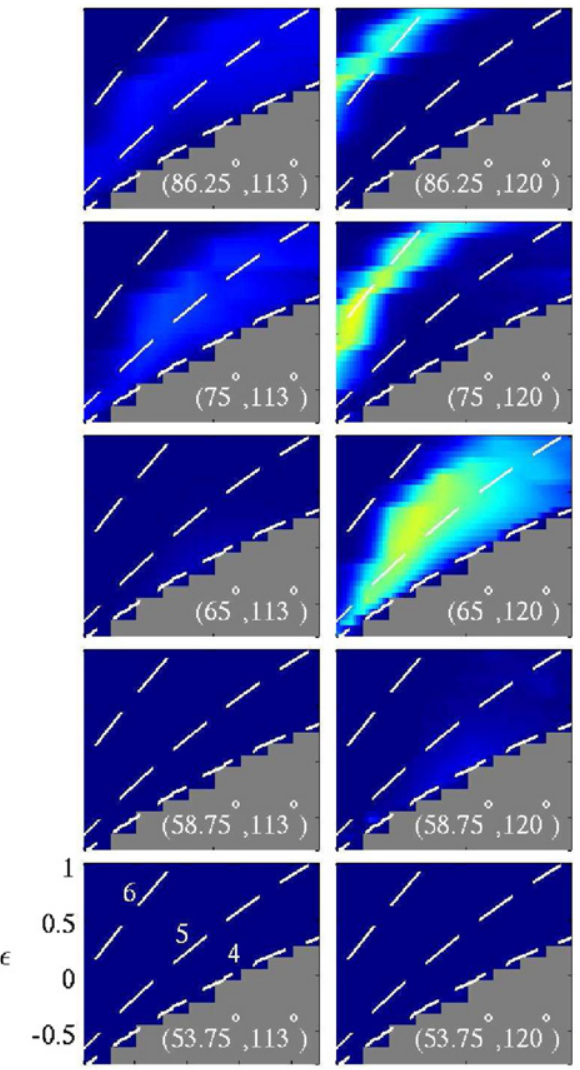

$\begin{array}{lllll}0 & 0.2 & 0.4 & 0.6 & 0.8\end{array}$

$a_{*}$
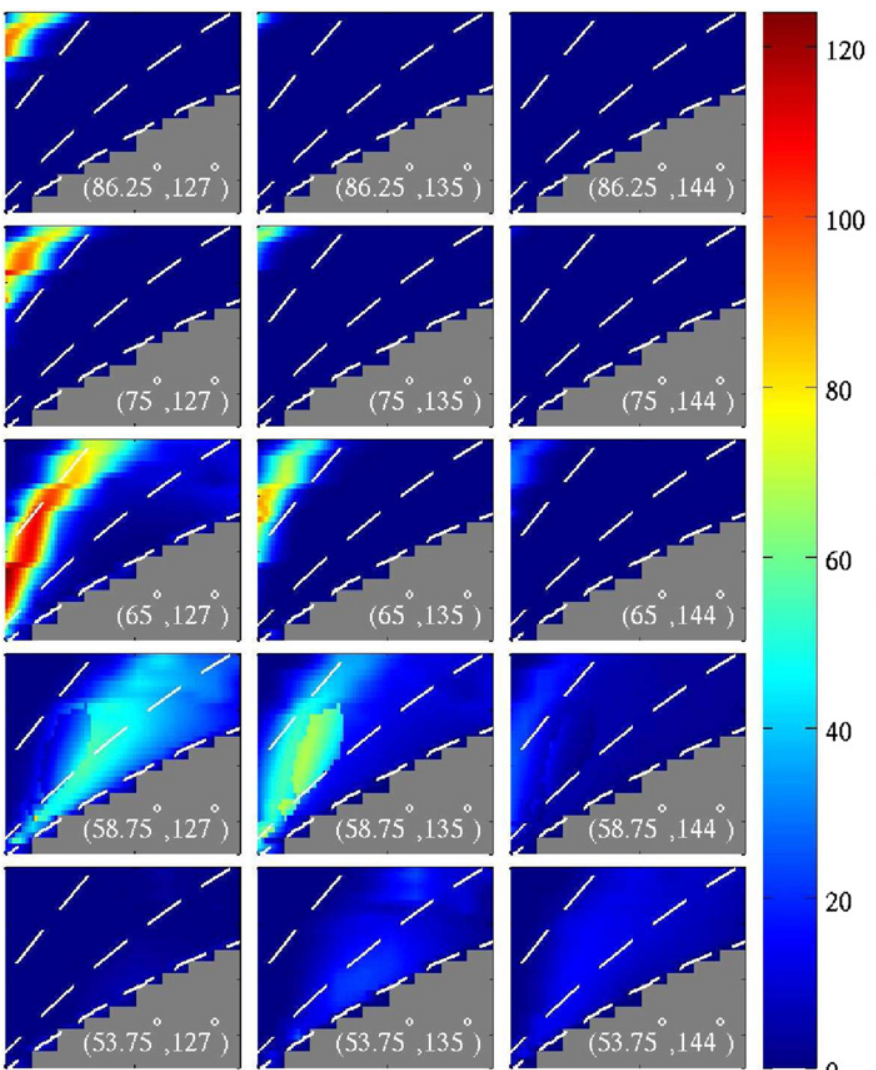

$\frac{3}{2}$

40

20

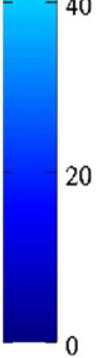

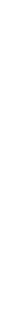

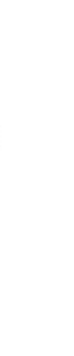

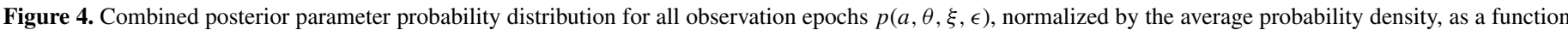

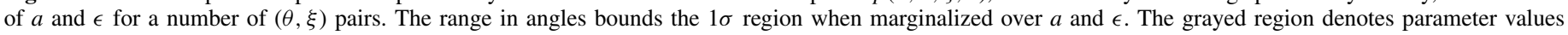

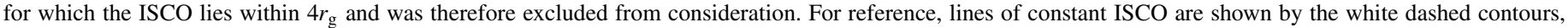
corresponding to $6 r_{\mathrm{g}}, 5 r_{\mathrm{g}}$, and $4 r_{\mathrm{g}}$ (top to bottom).

(A color version of this figure is available in the online journal.)

roughly $6 r_{\mathrm{g}}$, corresponding to the ISCO of a slightly modified Schwarzschild black hole with a value of the quadrupolar deviation parameter $\epsilon=-0.2_{-0.6}^{+1.2}$ and values of the inclination $\theta=65_{-12^{\circ}}^{\circ}$ and position angle $\xi=127^{\circ}{ }_{-14^{\circ}}^{\circ}$. Quoted are the bounds on the $1 \sigma$ envelope within the full four-dimensional parameter space, although these are potentially misleading since some of the parameters are significantly correlated (see the following sections).

The fine degree of the distinctions between images being made by the present EHT data is apparent in Figure 5. There the most probable image (middle row) is compared to images with identical black hole spin but a different quadrupolar perturbation parameter such that the posterior probability density is reduced by roughly half. As anticipated, the variations in the black hole silhouette induce corresponding variations in the visibilities that persist after Galactic electron scattering broadens the image. The electron scatter broadening substantially reduces the signal associated with variations on small scales, suggesting that the main constraining power arises due to the change in the ISCO location and shadow shape on large scales. However, the overall flux normalization, fixed observationally by the spectral data, couples the dynamical impact of the spacetime on small scales (long baseline length) to those on large scales (small baseline length). Hence, it remains difficult to unambiguously identify the primary source of the constraint. Nevertheless, it is clear that structural differences do exist on baselines already accessible by EHT observations.

\subsection{Implications for Previous Studies}

A key outstanding issue regarding the analysis in Broderick et al. (2011b) is robustness: it is unclear how robust the conclusions for spin magnitude and direction are to variations in the disk model and/or spacetime structure. Here we attempt to address this issue, using the quasi-Kerr metric as a proxy for just such a modification and assessing the associated sensitivity of the spin parameter estimates. Of particular relevance is the fact that nonzero values of the parameter $\epsilon$ probe larger ISCO radii, approximating the retrograde disk scenario, which heretofore has been neglected on dynamical grounds.

Despite the above concerns, it appears that the estimates of the spin orientation are reasonably robust. As seen in the left panel of Figure 6, the inclination is only weakly correlated with $\epsilon$. Most importantly, the lower limit on the inclination, immediately relevant for the ability to image a silhouette and the potential for dynamical lensing signals, is independent of the quadrupolar perturbation. This is independent of the prior placed on $\theta$; that is, choosing a flat prior on $\theta$ instead of the isotropic prior employed does not make a significant difference in the lower limit on the inclination. The position angle (center panel of Figure 6) is also nearly independent of $\epsilon$, with similar conclusions holding. The result is that the posterior probability distribution of the spin orientation (right panel of Figure 6) is tightly constrained despite the additional freedom afforded by the spacetime perturbation. Hence, the inferred spin orientation 
$\epsilon$
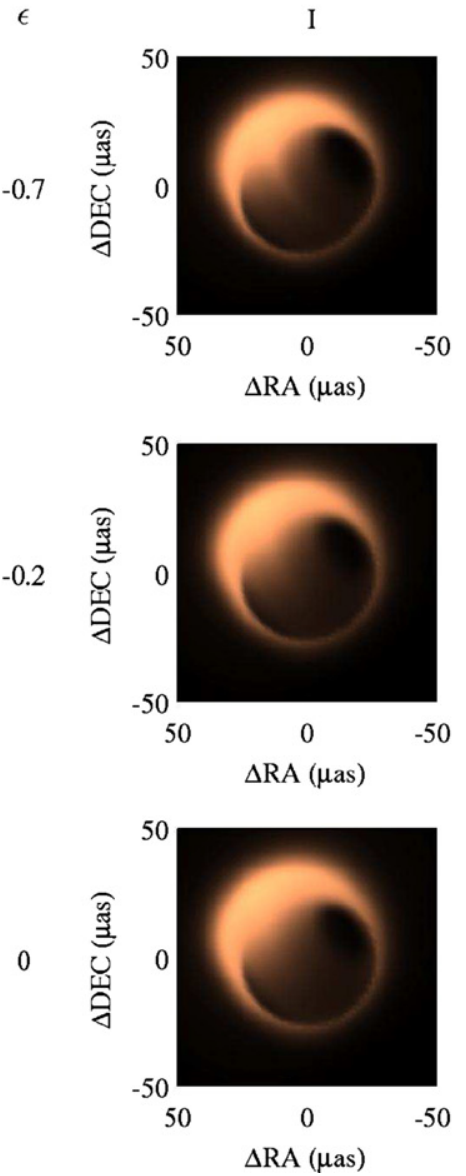

$|\mathrm{V}|$
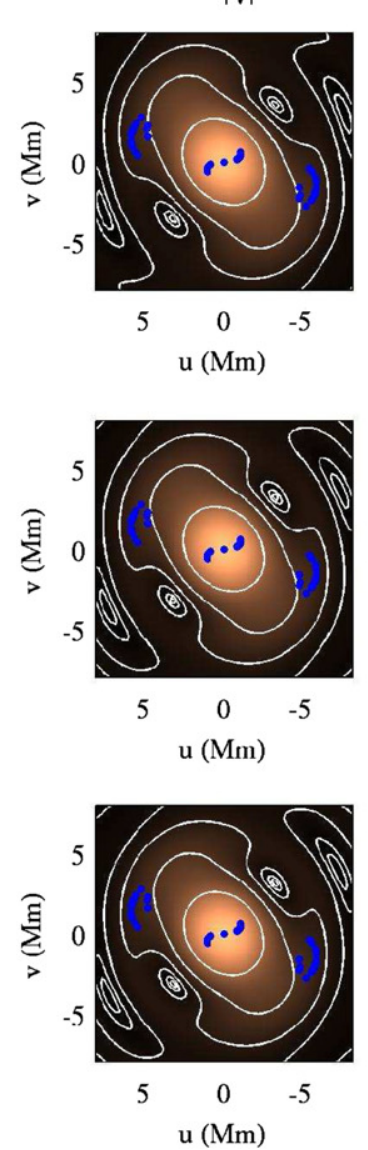
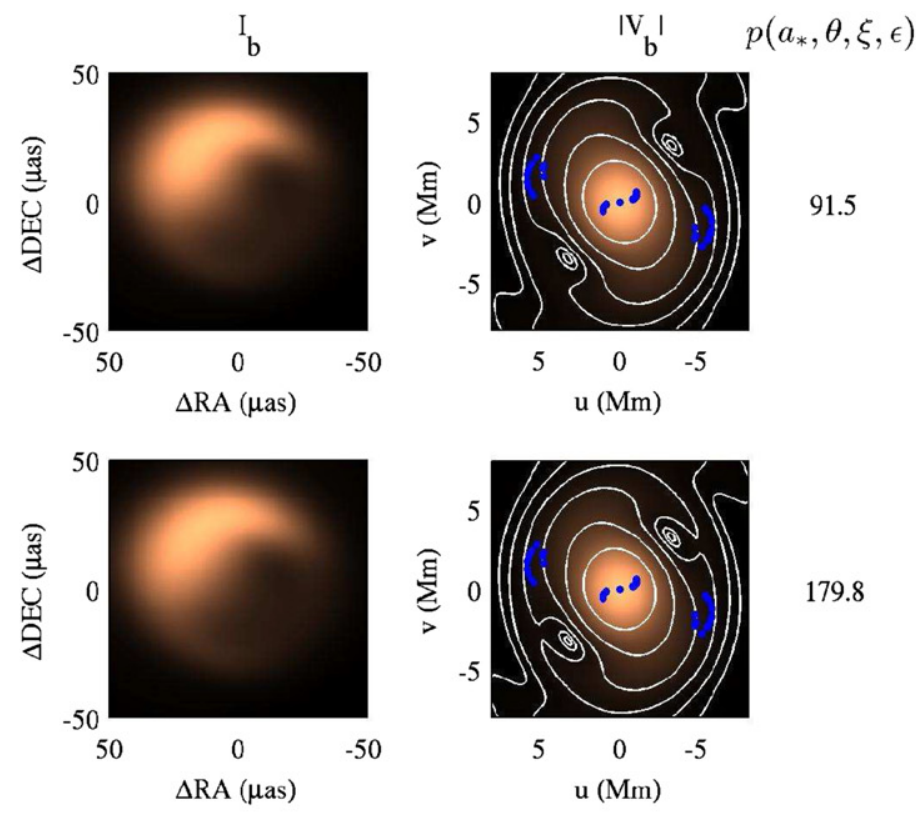

179.8
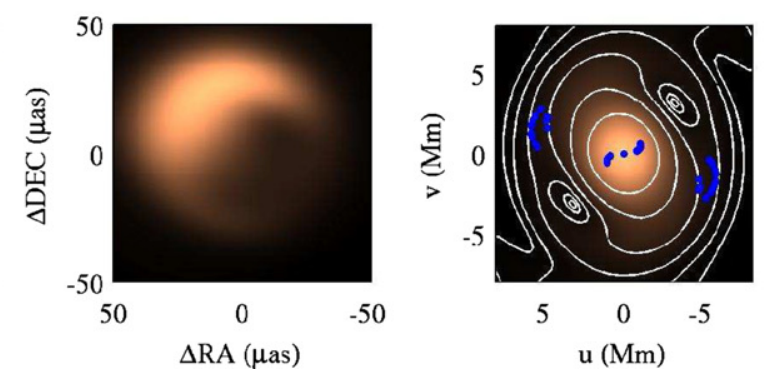

92.4

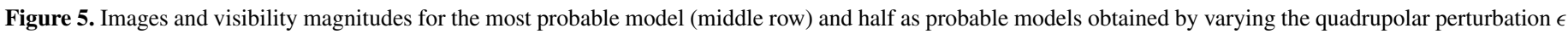

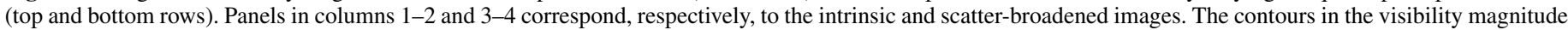

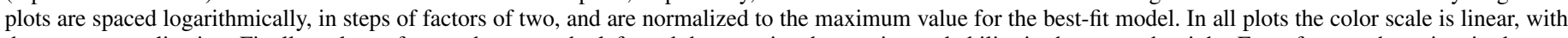

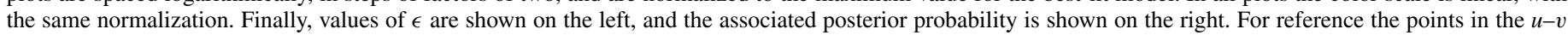
plane at which EHT data exist are shown by the blue points in the visibility magnitude plots.

(A color version of this figure is available in the online journal.)
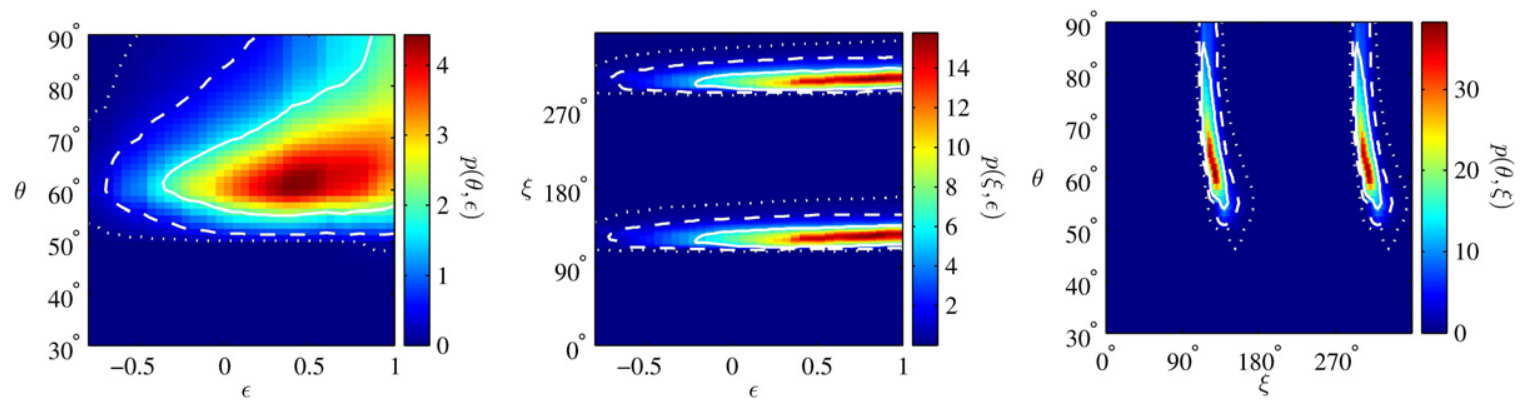

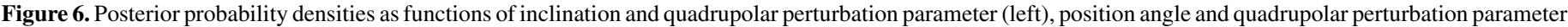

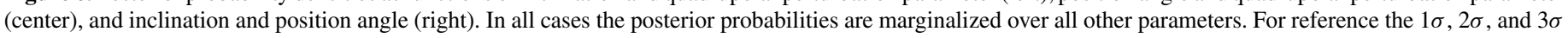
contours are shown by the solid, dashed, and dotted white lines, respectively.

(A color version of this figure is available in the online journal.)

(or in the case of vanishing spin, disk orientation) is robust to the sorts of perturbations considered here.

The implied impact on the spin magnitude depends somewhat on the precise quantity being measured. In Figure 4, the high probability density regions lie roughly along lines of fixed ISCO, implying that the primary quantity being measured is not the spin magnitude per se, but the dynamics of spacetime orbits and their subsequent effect on the accretion flow. As a result, $a$ and $\epsilon$ appear to be substantially correlated, while the ISCO is relatively robustly determined. As seen in Figure 7, the radius of the ISCO is localized near $6 r_{\mathrm{g}}$, falling rapidly at both substantially smaller and larger values. For reference, the associated spin parameter for a Kerr spacetime is shown on the top axis. Assuming that the variation of the ISCO is the dominant way in which spin enters into the EHT constraints, it implies a spin magnitude limit of $a_{*}=0.05_{-0.15}^{+0.27}$, which is comparable to the marginalized limits obtained in Broderick et al. (2011b).

The rapid decline in probability density at ISCO larger than $6 r_{\mathrm{g}}$ provides an indirect justification for the neglect of retrograde accretion flows. These were previously ignored because of the 


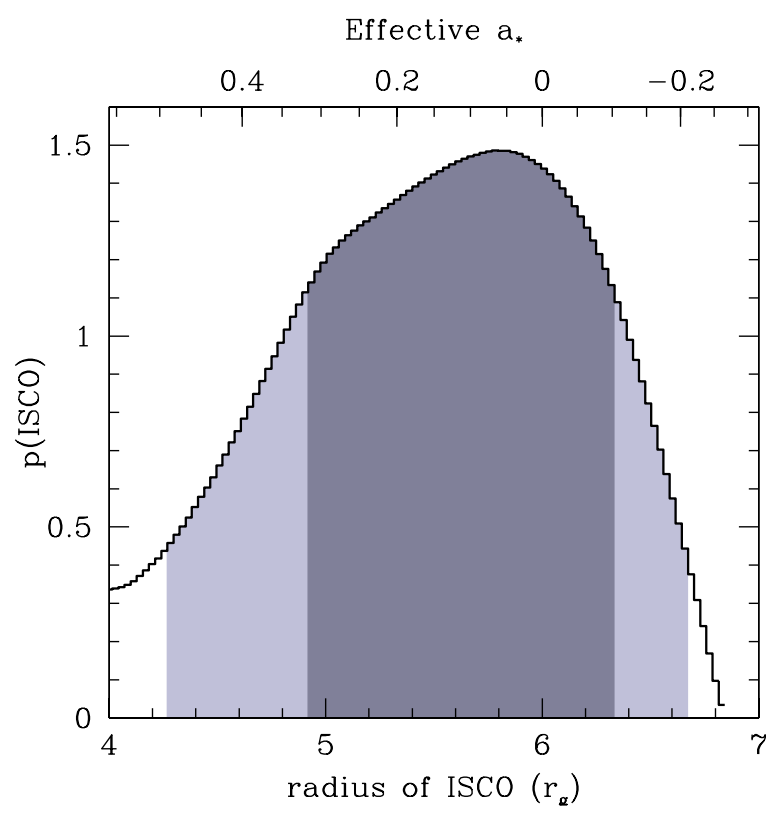

Figure 7. Posterior probability density as a function of the ISCO radius (in $r_{\mathrm{g}}$ ), marginalized over all other quantities. Dark and light gray shaded regions denote the $1 \sigma$ and $2 \sigma$ intervals, respectively. For reference, the inferred dimensionless spin magnitude for a Kerr black hole is shown on the top axis. The inclination and spin orientation angles depend only very weakly on the deviation parameter.

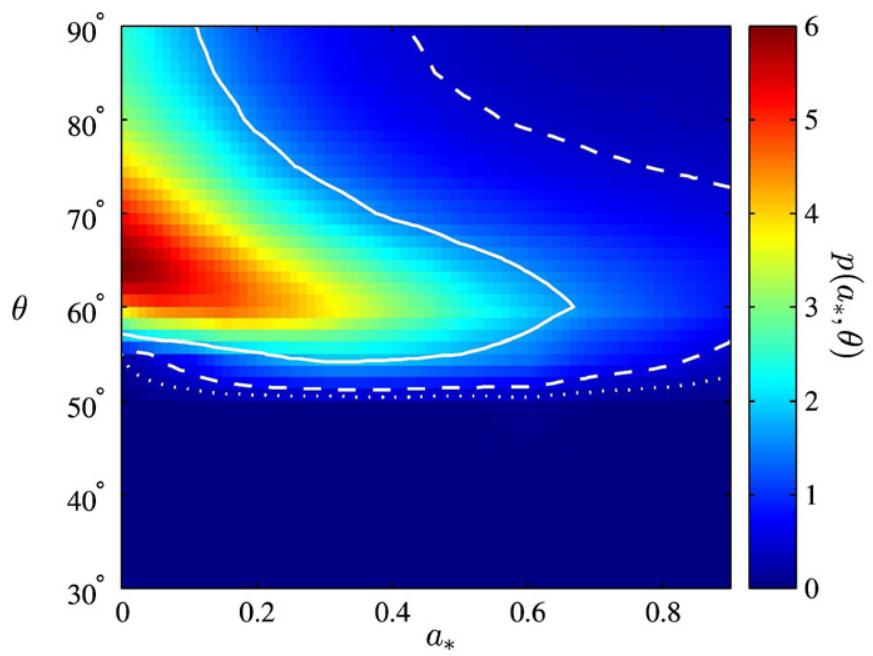

Figure 8. Posterior probability density as a function of dimensionless spin magnitude and inclination, marginalized over all other quantities. Solid, dashed, and dotted white lines show the $1 \sigma, 2 \sigma$, and $3 \sigma$ confidence contours, respectively.

(A color version of this figure is available in the online journal.)

dramatic dynamical consequences associated with the interplay between the Lense-Thirring torques and the orbital motion (Fragile et al. 2007), leading to concerns that stationary models of retrograde accretion flows were intrinsically inconsistent. However, insofar as the primary impact of the retrograde orbital motion is to increase the ISCO, Figure 7 argues empirically against retrograde accretion flow models and thus for low and positive spin values.

Despite the strong correlation between $a$ and $\epsilon$, on marginalization over the quadrupolar perturbation, the inferred spin magnitude is also reasonably robust. This may be seen explicitly in Figure 8, which shows the posterior probability distribution of spin magnitude and inclination, from which we find $a_{*}=0^{+0.7}$. This is directly comparable to Figure 4(b) in Broderick et al. (2011a), to which it is similar. While it does exhibit a modest

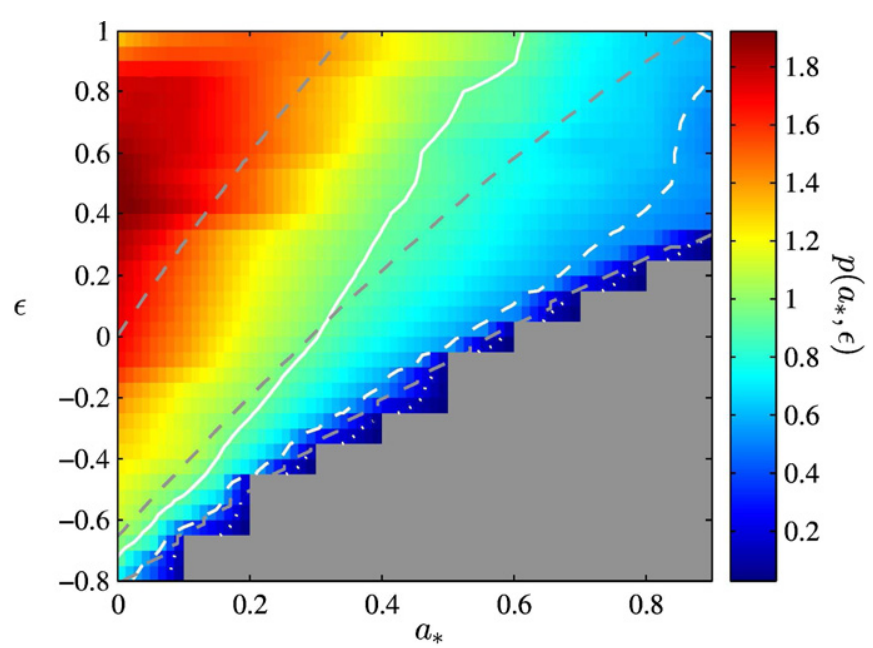

Figure 9. Posterior probability density as a function of dimensionless spin magnitude and quadrupolar perturbation, marginalized over all other quantities. The solid, dashed, and dotted lines show the $1 \sigma, 2 \sigma$, and $3 \sigma$ confidence regions, respectively. Lines of constant ISCO are shown by the dashed gray lines, corresponding to $6 r_{\mathrm{g}}, 5 r_{\mathrm{g}}$, and $4 r_{\mathrm{g}}$ from top to bottom. The gray region in the lower right is parameter values excluded by the condition that the ISCO lie outside of $4 r_{\mathrm{g}}$.

(A color version of this figure is available in the online journal.)

reduction in the small $a_{*}-\theta$ correlation, likely because of the limiting of our present analysis to $a_{*}<0.9$ and artifacts associated with the adaptive refinement scheme, the high-probability structures are completely consistent.

Quantitatively, this depends on the prior on $\epsilon$ that is assumed. The flat prior on $\epsilon$ employed here, combined with the restriction on the ISCO we have adopted, effectively results in a downweighting of $\epsilon \lesssim 0.3$, for which portions of the $a-\epsilon$ parameter space are excluded. Adopting a uniform prior on $\epsilon$, i.e., choosing the prior such that after marginalizing the prior over $a$ the resulting prior probability density in $\epsilon$ is flat, up-weights small $\epsilon$ and thus small spins. Thus, the posterior probability distribution shown in Figure 8 represents a rather pessimistic estimate of the limits on the spin magnitude. Its similarity to previous analyses suggests that efforts to constrain the spin magnitude are insensitive to even large spacetime perturbations.

\subsection{Limits on Deviations from the Kerr Metric}

While the high probability density regions lie roughly along contours of constant ISCO radius, the probability density is not uniformly distributed in $\epsilon$. Indeed, this is partly responsible for the robustness of the spin magnitude limits described in the preceding section. It also suggests that it may be possible to constrain $\epsilon$ from the current data.

The posterior probability distribution in spin magnitude and quadrupolar perturbation is shown in Figure 9. While a modest concentration does exist at positive $\epsilon$ and small $a$, the $2 \sigma$ confidence region extends essentially over the entire $a-\epsilon$ parameter space considered. Thus, as yet, there is little that can be said regarding the value of $\epsilon$ in a prior-independent manner. Nevertheless, the strong constraints apparent in the full parameter space (Figure 4) suggest that such limits will be forthcoming. We will address the strength of future limits from the EHT elsewhere.

\section{CONCLUSIONS}

We have constructed the first simulated images of an RIAF around a supermassive black hole described by a quasi-Kerr 
metric, which deviates from the Kerr metric in that it contains an independent quadrupole moment. Millimeter-wavelength VLBI observations are capable of constraining the magnitude of the quadrupolar deviations from the Kerr metric and therefore violations of the no-hair theorem. This remains true after folding in the impact of realistic accretion flow and radiative transfer models. The constraining capability arises due to both the modification of the null geodesic structure of the spacetime, and therefore the shape of the black hole shadow, and the impact on the dynamics of the accretion flow itself. It remains unclear to what degree these may be explicitly separated, although current EHT observations are already capable of distinguishing surprisingly similar images. Unfortunately, the current published EHT data do not place a strong limit on the quadrupole deviation $\epsilon$ yet. We will address the strength of upcoming observations elsewhere.

Despite the introduction of an additional model parameter, the result for the black hole spin as inferred from previous studies is robust. This is especially true for the orientation, which is largely insensitive to the quadrupolar distortion. Even with the additional freedom, the best-fit inclination and position angles are $\theta=65_{-11^{\circ}}^{\circ}$ and $\xi=127^{\circ}{ }_{-14^{\circ}}^{\circ}$ (up to the $180^{\circ}$ degeneracy), consistent with previously obtained values. This suggests that the orientation of the spin (or accretion flow angular momentum) is well constrained despite the substantial astrophysical uncertainties that remain (D. Psaltis et al., in preparation).

The spin magnitude and quadrupolar perturbation are correlated such that the ISCO is limited to $\left(5.8_{-1.1}^{+0.7}\right) r_{\mathrm{g}}$. Within the context of the Kerr metric, this argues against the need to consider retrograde accretion flows, previously disregarded on dynamical grounds. If translated into a limit on the spin magnitude using the ISCO-spin relationship of the Kerr spacetime, it implies $a_{*}=0.05_{-0.15}^{+0.27}$.

Despite this correlation, the posterior probability distribution of the spin magnitude is quite similar to that found in previous analyses of the EHT data, implying $a_{*}=0^{+0.7}$. While this conclusion depends quantitatively on the priors assumed for the quadrupolar perturbation, it is qualitatively true for all reasonable choices.

Here we restricted our analysis to spacetimes for which the ISCO lies at a radius greater than or equal to $4 r_{\mathrm{g}}$ in order to avoid potential pathologies of the quasi-Kerr arising at smaller radii. Since Sgr A* appears to be only slowly spinning, this may be sufficient for this source. Typically, however, the most dramatic effects due to deviations from the Kerr metric manifest at small radii, accessible to accretion flows around rapidly spinning black holes. Generating images of RIAFs about these black holes requires the use of Kerr-like metrics that are free of pathological regions (e.g., Johannsen \& Psaltis 2011a; Johannsen 2013b). We will present such images in future work.

A.E.B. received financial support from Perimeter Institute for Theoretical Physics and the Natural Sciences and Engineering Research Council of Canada through a Discovery Grant. T.J. was supported by a CITA National Fellowship at the University of Waterloo and in part by Perimeter Institute for Theoretical Physics. Research at Perimeter Institute is supported by the government of Canada through Industry Canada and by the province of Ontario through the Ministry of Research and Innovation. A.L. was supported in part by NSF grants AST0907890, AST-0807843, and AST-0905844 and NASA grants
NNX08AL43G and NNA09DB30A. D.P. was supported by an NSF CAREER award NSF 0746549.

\section{APPENDIX A}

\section{ANALYTICAL FLUX NORMALIZATION LIKELIHOOD MARGINALIZATION/MAXIMIZATION}

Each observational epoch has an unknown flux normalization, assumed to arise from moderate fluctuations in the accretion rate, which to lowest order just rescale the intensities across an image. Here we obtain analytical expressions in the presence of Gaussian errors and a flat prior on this normalization $V_{00}$ for the marginalized and maximized likelihoods.

In practice, for each observational epoch $E$, we have a set of observed visibilities $V_{j, E}$ at the points $\left(u_{j}, v_{j}\right)$, with some error estimates $\sigma_{j, E}$. Similarly, we have a set of model visibilities $V_{00, E} \hat{V}_{j}(\mathbf{p})$, where $\mathbf{p}$ are the various parameters that define the image model beyond the observational epoch-dependent flux normalizations $V_{00, E}$. Then the associated likelihood is

$$
L\left(V_{00,1}, V_{00,2}, \ldots ; \mathbf{p}\right)=N \exp \left[\sum_{E, j} \frac{\left(V_{j, E}-V_{00, E} \hat{V}_{j}\right)^{2}}{2 \sigma_{j, E}^{2}}\right] \text {, }
$$

where $N$ is a fixed normalization. Without loss of generality, we can define $y_{j, E} \equiv V_{j, E} / \sigma_{j, E}$ and $\hat{y}_{j} \equiv \hat{V}_{j} / \sigma_{j, E}$ and thus treat only unit-normalized distributions, i.e.,

$$
L=N \exp \left[\frac{1}{2} \sum_{E, j}\left(y_{j, E}-V_{00, E} \hat{y}_{j}\right)^{2}\right] .
$$

In the above and henceforth, for simplicity we will suppress all functional dependencies.

The likelihood defined in Equation (A2) is maximized relative to the flux normalization when

$$
\frac{\partial L}{\partial V_{00, E}}=\left[\sum_{j}\left(y_{j, E}-V_{00, E} \hat{y}_{j}\right) \hat{y}_{j}\right] L=0,
$$

and thus we obtain the standard result

$$
V_{00, E}^{M}=\frac{\sum_{J} y_{j, E} \hat{y}_{j}}{\sum_{J} \hat{y}_{j}^{2}} .
$$

Inserting this back into the likelihood and simplifying gives the desired analytical form for the maximum likelihood:

$$
L^{M}=N \prod_{E} \exp \left[\frac{\left(\sum_{j} y_{j, E}^{2}\right)\left(\sum_{j} \hat{y}_{j}^{2}\right)-\left(\sum_{j} y_{j, E} \hat{y}_{j}\right)^{2}}{2 \sum_{j} \hat{y}_{j}^{2}}\right] \text {. }
$$

On the other hand, the marginalized likelihood is defined by

$$
\begin{aligned}
\bar{L}= & \int d V_{00,1} d V_{00,2} \ldots \pi\left(V_{00,1}\right) \pi\left(V_{00,2}\right) \ldots L \\
= & L^{M} \prod_{E} \int d V_{00, E} \\
& \times \Pi\left(V_{00, E}\right) \exp \left[-\left(\sum_{j} \frac{\hat{y}_{j}^{2}}{2}\right)\left(V_{00, E}-V_{00, E}^{M}\right)^{2}\right],
\end{aligned}
$$


where $\Pi\left(V_{00, E}\right)$ is the prior on $V_{00, E}$. If $\Pi\left(V_{00, E}\right)$ varies slowly over variations in $V_{00, E}$ that are much wider than the peak of the exponential in the final integral, we may approximate it as flat and extending from $V_{00, E}=-\infty$ to $\infty$. Thus, the average value of $V_{00, E}$ is simply $V_{00, E}^{M}$, and the resulting marginalized likelihood is

$$
\bar{L}=L^{M} \prod_{E}\left(\frac{2 \pi}{\sum_{j} \hat{y}_{j}^{2}}\right)^{1 / 2} \Pi\left(V_{00, E}^{M}\right),
$$

where $\Pi\left(V_{00, E}^{M}\right)$ is simply a constant and may be neglected in further parameter estimation.

\section{APPENDIX B}

\section{MARGINALIZED LIKELIHOODS AND POSTERIOR PROBABILITY CONSTRUCTION}

The desired analysis product is essentially a posterior probability distribution, marginalized over various nuisance parameters (in the present context, the epoch-dependent flux normalizations). Here we show that these are trivially related to the marginalized likelihoods.

Consider a model with a set of $n$ interesting parameters, $\mathbf{p}$, and $m$ nuisance parameters, $\mathbf{q}$, for which we have likelihoods $L(\mathbf{p}, \mathbf{q})$ with priors $\Pi(\mathbf{p}, \mathbf{q})$. Then the posterior probability density is given by Bayes' theorem by

$$
p(\mathbf{p}, \mathbf{q})=\frac{L(\mathbf{p}, \mathbf{q}) \Pi(\mathbf{p}, \mathbf{q})}{\int d^{n} p d^{m} q L(\mathbf{p}, \mathbf{q}) \Pi(\mathbf{p}, \mathbf{q})} .
$$

After marginalizing over the nuisance parameters, we obtain the desired probability density,

$$
p(\mathbf{p})=\int d^{m} q p(\mathbf{p}, \mathbf{q})=\frac{\int d^{m} q L(\mathbf{p}, \mathbf{q}) \Pi(\mathbf{p}, \mathbf{q})}{\int d^{n} p d^{m} q L(\mathbf{p}, \mathbf{q}) \Pi(\mathbf{p}, \mathbf{q})} .
$$

If the priors on the physical and nuisance parameters are separable, i.e., $\Pi(\mathbf{p}, \mathbf{q})=\Pi_{p}(\mathbf{p}) \Pi_{q}(\mathbf{q})$, then this may be written as

$$
p(\mathbf{p})=\frac{\bar{L}(\mathbf{p}) \Pi_{p}(\mathbf{p})}{\int d^{n} p \bar{L}(\mathbf{p}) \Pi_{p}(\mathbf{p})},
$$

where

$$
\bar{L}(\mathbf{p}) \equiv \int d^{m} q L(\mathbf{p}, \mathbf{q}) \Pi_{q}(\mathbf{q})
$$

are the marginalized likelihoods. Thus, the marginalization over the nuisance parameters may be performed during the construction of the likelihoods themselves, as in Appendix A.

\section{REFERENCES}

Amarilla, L., \& Eiroa, E. F. 2012, PhRvD, 85, 064019

Amarilla, L., Eiroa, E. F., \& Giribet, G. 2010, PhRvD, 81, 124045

Bambi, C. 2012a, ApJ, 761, 174

Bambi, C. 2012b, JCAP, 09, 014

Bambi, C. 2013, PhRvD, 87, 023007

Bambi, C., \& Barausse, E. 2011, ApJ, 731, 121

Bambi, C., Caravelli, F., \& Modesto, L. 2012, PhLB, 711, 10

Bambi, C., \& Yoshida, N. 2010, CQGra, 27, 205006

Blandford, R. D., \& Begelman, M. C. 1999, MNRAS, 303, L1

Bower, G. C., Goss, W. M., Falcke, H., Backer, D. C., \& Lithwick, Y. 2006, ApJL, $648, \mathrm{~L} 127$
Broderick, A., \& Blandford, R. 2004, MNRAS, 349, 994

Broderick, A. E. 2006, MNRAS, 366, L10

Broderick, A. E., Fish, V. L., Doeleman, S. S., \& Loeb, A. 2009, ApJ, 697, 45

Broderick, A. E., Fish, V. L., Doeleman, S. S., \& Loeb, A. 2011a, ApJ, 738, 38

Broderick, A. E., Fish, V. L., Doeleman, S. S., \& Loeb, A. 2011b, ApJ, 735,110

Broderick, A. E., \& Loeb, A. 2006a, ApJL, 636, L109

Broderick, A. E., \& Loeb, A. 2006b, MNRAS, 367, 905

Collins, N. A., \& Hughes, S. A. 2004, PhRvD, 69, 124022

Dexter, J., Agol, E., Fragile, P. C., \& McKinney, J. C. 2010, ApJ, 717, 1092

Dexter, J., \& Fragile, P. C. 2013, MNRAS, 432, 2252

Doeleman, S. S., Fish, V. L., Schenck, D. E., et al. 2012, Sci, 338, 355

Doeleman, S. S., Weintroub, J., Rogers, A. E. E., et al. 2008, Natur, 455, 78

Fabian, A. C. 2012, ARA\&A, 50, 455

Falcke, H., \& Markoff, S. 2000, A\&A, 362, 113

Fish, V. L., Alef, W., Anderson, J., et al. 2013, arXiv:1309.3519

Fish, V. L., Doeleman, S. S., Beaudoin, C., et al. 2011, ApJL, 727, L36

Fragile, P. C., Blaes, O. M., Anninos, P., \& Salmonson, J. D. 2007, ApJ, 668,417

Furlanetto, S. R., \& Loeb, A. 2001, ApJ, 556, 619

Gair, J. R., Vallisneri, M., Larson, S. L., \& Baker, J. G. 2013, LRR, 16, 7

Geroch, J. 1970, JMP, 11, 2580

Ghez, A. M., Salim, S., Weinberg, N. N., et al. 2008, ApJ, 689, 1044

Gierliński, M., Middleton, M., Ward, M., \& Done, C. 2008, Natur, 455, 369

Gillessen, S., Eisenhauer, F., Fritz, T. K., et al. 2009a, ApJL, 707, L114

Gillessen, S., Eisenhauer, F., Trippe, S., et al. 2009b, ApJ, 692, 1075

Glampedakis, K., \& Babak, S. 2006, CQGra, 23, 4167

Hansen, R. O. 1974, JMP, 15, 46

Hartle, J. B. 1967, ApJ, 150, 1005

Hartle, J. B., \& Thorne, K. S. 1968, ApJ, 153, 807

Heusler, M. 1996, Black Hole Uniqueness Theorems (Cambridge: Cambridge Univ. Press)

Huang, L., Takahashi, R., \& Shen, Z. 2009, ApJ, 706, 960

Johannsen, T. 2013a, ApJ, 777, 170

Johannsen, T. 2013b, PhRvD, 88, 044002

Johannsen, T. 2013c, PhRvD, 87, 124017

Johannsen, T., \& Psaltis, D. 2010a, ApJ, 716, 187

Johannsen, T., \& Psaltis, D. 2010b, ApJ, 718, 446

Johannsen, T., \& Psaltis, D. 2011a, PhRvD, 83, 124015

Johannsen, T., \& Psaltis, D. 2011b, ApJ, 726, 11

Johannsen, T., \& Psaltis, D. 2013, ApJ, 773, 57

Jones, T. W., \& O’Dell, S. L. 1977, ApJ, 214, 522

Kennea, J. A., Burrows, D. N., Kouveliotou, C., et al. 2013, ApJL, 770, L24

Krawczynski, H. 2012, ApJ, 754, 133

Liu, K., Wex, N., Kramer, M., Cordes, J. M., \& Lazio, T. J. W. 2012, ApJ, 747,1

Loeb, A., \& Waxman, E. 2007, JCAP, 03, 011

Manko, V. S., \& Novikov, I. D. 1992, CQGra, 9, 2477

McClintock, J. E., Narayan, R., \& Steiner, J. F. 2013, arXiv:1303.1583

Meier, D. L. 2012, Black Hole Astrophysics: The Engine Paradigm (Berlin: Springer)

Merritt, D., Alexander, T., Mikkola, S., \& Will, C. M. 2010, PhRvD, 81, 062002

Mészáros, P. 2006, RPPh, 69, 2259

Myrzakulov, R., Sebastiani, L., \& Zerbini, S. 2013, IJMPD, 22, 30017

Narayan, R. 2005, NJPh, 7, 199

Narayan, R., Mahadevan, R., Grindlay, J. E., Popham, R. G., \& Gammie, C. 1998, ApJ, 492, 554

Özel, F., Psaltis, D., \& Narayan, R. 2000, ApJ, 541, 234

Petrosian, V., \& McTiernan, J. M. 1983, PhFl, 26, 3023

Psaltis, D. 2008, LRR, 11, 9

Reis, R. C., Miller, J. M., Reynolds, M. T., et al. 2012, Sci, 337, 949

Remillard, R. A., \& McClintock, J. E. 2006, ARA\&A, 44, 49

Reynolds, C. S. 2013, arXiv:1302.3260

Ryan, F. D. 1995, PhRvD, 52, 5707

Shcherbakov, R. V., Penna, R. F., \& McKinney, J. C. 2012, ApJ, 755, 133

Thorne, K. S. 1980, RvMP, 52, 299

van der Klis, M. 2006, in Compact Stellar X-ray Sources, ed. W. H. G. Lewin \& M. van der Klis (Cambridge: Cambridge Univ. Press), 39

Vietri, M. 2008, Foundations of High-Energy Astrophysics (Chicago, IL: Univ. Chicago Press)

Vigeland, S., Yunes, N., \& Stein, L. C. 2011, PhRvD, 83, 104027

Vigeland, S. J., \& Hughes, S. A. 2010, PhRvD, 81, 024030

Wex, N., \& Kopeikin, S. M. 1999, ApJ, 514, 388

Will, C. M. 2008, ApJL, 674, L25

Yuan, F., Quataert, E., \& Narayan, R. 2003, ApJ, 598, 301 